\title{
Moduli of extensions of holomorphic bundles on Kähler manifolds
}

\author{
Georgios Daskalopoulos ${ }^{1}$, Karen Uhlenbeck \\ AND RICHARD WENTWORTH ${ }^{2}$
}

\begin{abstract}
We introduce in this paper a moduli space parametrizing extensions of holomorphic bundles on Kähler manifolds. A notion of stability for extensions is given generalizing the definition for bundles, and an existence theorem for special metrics on stable extensions giving solutions to an extended Hermitian-Einstein equation is proven. We describe the construction of the moduli space of solutions to these equations via gauge theory techniques and, in the case of algebraic manifolds, we alternatively construct the moduli via geometric invariant theory.
\end{abstract}

\section{Introduction.}

In this paper we introduce a moduli space parametrizing extensions of holomorphic bundles on Kähler manifolds. The equations describing the moduli space are closely related to the harmonic map equations from Kähler manifolds to Grassmannians and flag varieties and were studied, in the case of Riemann surfaces, by the second author in connection with harmonic maps into Lie groups and coadjoint orbits (cf. [U1-2], [Hi] for further details).

For holomorphic bundles $E_{1}^{D_{1}^{\prime \prime}}, E_{2}^{D_{2}^{\prime \prime}}$ on a Kähler manifold $X$ and a harmonic $(0,1)$ form $\varphi$ with values in $\operatorname{Hom}\left(E_{2}, E_{1}\right)$ we form the equations (see $\S 1.1$ for details)

$$
\left\{\begin{array}{c}
\sqrt{-1} \Lambda\left(F_{D_{1}^{\prime \prime}, H_{1}}-\varphi \wedge \varphi^{*}\right)=\tau_{1} \mathbf{I}_{1} \\
\sqrt{-1} \Lambda\left(F_{D_{2}^{\prime \prime}, H_{2}}-\varphi^{*} \wedge \varphi\right)=\tau_{2} \mathbf{I}_{2}
\end{array}\right.
$$

for hermitian metrics $H_{1}, H_{2}$ on $E_{1}, E_{2}$. In the above, $\tau_{1}, \tau_{2}$ are real parameters. The purpose of this paper is to describe the space of equivalence

\footnotetext{
${ }^{1}$ Supported in part by NSF Grant DMS-9504297

${ }^{2}$ Supported in part by NSF Grant DMS-9503635
} 
classes of solutions to (0.1). By using $\varphi$ as a second fundamental form for the split holomorphic structure on $E=E_{1} \oplus E_{2}$ we obtain a holomorphic structure $D^{\prime \prime}$ - if $H_{1}, H_{2}$ satisfy (0.1), then the induced hermitian metric $H$ on $E$ satisfies

$$
\begin{cases}\sqrt{-1} \Lambda F_{D^{\prime \prime}, H} & =\tau_{1} \pi_{H}+\tau_{2}\left(\mathbf{I}-\pi_{H}\right) \\ \left(\mathbf{I}-\pi_{H}\right) D^{\prime \prime} \pi_{H} & =0 .\end{cases}
$$

Here, $\pi_{H}$ is the projection onto $E_{1}$ with respect to the metric $H$.

In $\S 1$ we prove that $(0.1)$ and $(0.2)$ are equivalent (see Proposition 1.5). The advantage of (0.2) is that it can interpreted as a moment map for the action of the full group of gauge transformations of $E$. In order to achieve a direct moment map interpretation for (0.1) it is necessary to restrict to a subgroup of the gauge group. This causes difficulty in the application of the infinite dimensional version of the Kempf-Ness theorem due to a lack of convexity. These problems are circumvented by reformulating the equations as (0.2).

In $\S 2$ we describe the stability condition for holomorphic "extension" pairs $\left(E_{1}, E\right)$ which is associated to solutions of $(0.2)$. We formulate the stability for a torsion-free sheaf $E$ and a subsheaf $E_{1}$. Equivalently, we describe the stability for quotients $E \rightarrow F \rightarrow 0$ (see Proposition 2.8). An important point is that for algebraic manifolds we have two notions of stability: Mumford-Takemoto or slope stability, and Gieseker stability. Both depend on a choice of real parameter $\tau$ which is linearly related to the $\tau_{1}$ and $\tau_{2}$ parameters appearing in (0.2) (see (1.8) and (1.9)). It is interesting that Mumford and Gieseker stability are different notions, even in the case of Riemann surfaces.

In $\S 3$ we prove the Hitchin-Kobayashi correspondence between Mumford stable extension pairs $\left(E_{1}, E\right)$ and solutions to the equations (0.2) (see Theorem 3.1). Our proof is a combination of the arguments of Simpson [S1] and standard properties of the Grassmannian. One interesting aspect is the particular choice of Sobolev spaces which describe the "weak-subbundles" of [UY]. This permits a proof of the correspondence theorem for arbitrary saturated subsheaves $E_{1}$ rather than for only smooth subbundles.

In $\S 4$ we give a gauge-theoretic construction of the moduli space of extension pairs. We prove that generically it has the structure of a Kähler manifold (see Theorem 4.1). In $\S 5$ the moduli space is constructed via geometric invariant theory (or "GIT") for the case where $X$ is algebraic. From the point of view of algebraic geometry, it is more natural to work with quotient pairs. We prove that the moduli space of Gieseker stable quotients 
$E \rightarrow F \rightarrow 0$ where $E$ is torsion-free is a quasi-projective variety with a compactification given by adding in equivalence classes of Gieseker semistable quotients. The method closely follows that of Simpson [S2].

To conclude this introduction we would like to mention some questions related to our construction. One problem is to describe an analytic compactification of the moduli space of extension pairs in the case of complex dimension 1 and 2. Unfortunately, there is no natural candidate. One possibility is to relate such a compactification to the algebraic one in $\S 5$ in a manner similar to [Li] and [BDW].

A second problem is to study the behavior of the moduli spaces with respect to the parameter $\tau$. The picture should be similar to that found in [Th]; however, there is an extra complication coming from the non-compactness of the moduli spaces in the $\varphi$ direction. One motivation for this is the following: For certain values of $\tau$ the moduli spaces correspond to compactifications of the space of holomorphic maps from $X$ to a Grassmannian, at least when $X$ is simply connected. A concrete description of these birational equivalences may yield specific cohomological information about the space of holomorphic maps. Another application may be towards understanding the existence and structure of harmonic maps to Grassmannians (cf. [U1-2], [Hi]). This follows from the observation that the equations (0.1) make sense and are elliptic on Riemannian (possibly non-complex) four manifolds. These issues will be treated elsewhere.

After this paper was completed, we became aware of the article [T] where equations (1.1) are written for the case $\tau_{1}=\tau_{2}=\mu$, and we received the preprint $[\mathrm{BrGP}]$ where Theorem 3.1 is similarly proved.

Acknowledgment. We would like to thank T. Pantev for many useful discussions during the preparation of this manuscript.

\section{Formulation of the Equations.}

\subsection{Extended Hermitian-Einstein equations.}

Throughout the paper $X$ will be a compact Kähler manifold of complex dimension $n$ with Kähler form $\omega$. Let $E_{1}$ and $E_{2}$ be smooth, hermitian vector bundles on $X$ of ranks $R_{1}, R_{2}$, and $\omega$-degree $d_{1}, d_{2}$, respectively. We 
482 Georgios Daskalopoulos, Karen Uhlenbeck and Richard Wentworth

recall that the $\omega$-degree of a complex vector bundle $E$ is defined by

$$
\operatorname{deg} E=\int_{X} c_{1}(E) \wedge \omega^{n-1} .
$$

We also fix background hermitian metrics $K_{1}, K_{2}$ on the bundles $E_{1}, E_{2}$.

Given holomorphic structures $D_{1}^{\prime \prime}, D_{2}^{\prime \prime}$ on $E_{1}, E_{2}$, and

$$
\varphi \in \Omega^{0,1}\left(\operatorname{Hom}\left(E_{2}, E_{1}\right)\right),
$$

consider the equations

$$
\left\{\begin{array}{l}
\sqrt{-1} \Lambda\left(F_{D_{1}^{\prime \prime}, H_{1}}-\varphi \wedge \varphi^{*}\right)=\tau_{1} \mathbf{I}_{1} \\
\sqrt{-1} \Lambda\left(F_{D_{2}^{\prime \prime}, H_{2}}-\varphi^{*} \wedge \varphi\right)=\tau_{2} \mathbf{I}_{2} \\
\Delta^{\prime \prime} \varphi=0
\end{array}\right.
$$

for hermitian metrics $H_{1}, H_{2}$ on $E_{1}, E_{2}$, and real parameters $\tau_{1}, \tau_{2}$. In the above, $F_{D_{i}^{\prime \prime}, H_{i}}$ denotes the curvature of the unique hermitian connection compatible with $D_{i}^{\prime \prime}$ and $H_{i} ; \mathbf{I}_{i}$ denotes the identity endomorphism of $E_{i}$, $\Lambda$ contraction with $\omega, *$ the adjoint with respect to the metrics $H_{i}$ (i.e. $\left.\left\langle\varphi^{*} v, w\right\rangle_{H_{2}}=\langle v, \varphi w\rangle_{H_{1}}\right)$, and $\Delta^{\prime \prime}$ the Laplacian on $(0,1)$ forms with values in $\operatorname{Hom}\left(E_{2}, E_{1}\right)$. Notice that that $*$ defines a complex anti-linear isomorphism:

$$
\begin{array}{ll}
\Omega^{0,1}\left(\operatorname{Hom}\left(E_{2}, E_{1}\right)\right) & \longrightarrow \Omega^{1,0}\left(\operatorname{Hom}\left(E_{1}, E_{2}\right)\right) \\
\varphi & \longrightarrow \varphi^{*} .
\end{array}
$$

Hence, $\varphi \wedge \varphi^{*}$ and $\varphi^{*} \wedge \varphi$ are forms of type $(1,1)$.

Here is the motivation for equations (1.1) : By using $\varphi$ as the second fundamental form, we get a hermitain metric and hermitian connection on $E=E_{1} \oplus E_{2}$ from

$$
\begin{aligned}
D^{\prime \prime} & =D_{1}^{\prime \prime} \oplus D_{2}^{\prime \prime}+\left(\begin{array}{cc}
0 & \varphi \\
0 & 0
\end{array}\right) \\
H & =H_{1} \oplus H_{2} .
\end{aligned}
$$

If $D^{\prime \prime}$ is Hermitian-Einstein, i.e. if

$$
\sqrt{-1} \Lambda F_{D^{\prime \prime}, H}=\mu \mathbf{I},
$$

with $\mu$ constant, then the first two equations in (1.1) are satisfied for $\tau_{1}=$ $\tau_{2}=\mu$.

In this paper, we shall be mostly interested in the reformulation of (1.1) in terms of the connection on $E$. Perturbing the parameters $\tau_{1}$ and $\tau_{2}$ 
away from $\mu$ perturbs the Hermitian-Einstein equation (1.3). The result is expressed in terms of projections. Thus, let $\pi_{H}: E \rightarrow E$ denote the projection operator with respect to the metric $H$ onto $E_{1}$. Thus, $\pi_{H}=$ $\pi_{H}^{*}=\pi_{H}^{2}$, and $\pi_{H}(E)=E_{1}$. Now consider the equations

$$
\begin{cases}\sqrt{-1} \Lambda F_{D^{\prime \prime}, H} & =\tau_{1} \pi_{H}+\tau_{2} \pi_{H}^{\perp} \\ \pi_{H}^{\perp} D^{\prime \prime} \pi_{H} & =0 .\end{cases}
$$

where $\pi_{H}^{\perp}=\mathbf{I}-\pi_{H}$.

Proposition 1.5. Equations (1.1) and (1.4) are equivalent; i.e.

$$
\left(D_{1}^{\prime \prime}, D_{2}^{\prime \prime}, H_{1}, H_{2}, \varphi\right)
$$

satisfies (1.1) if and only if $\left(D^{\prime \prime}, H, \pi_{H}\right)$ satisfies (1.4).

Proof. Let $D_{i}$ denote the hermitian connections associated to the $D_{i}^{\prime \prime}$ and $H_{i}$, and let $D=D_{1} \oplus D_{2}$. According to (1.2),

$$
\begin{aligned}
& F_{D^{\prime \prime}, H}=F_{D}+D\left(\begin{array}{cc}
0 & \varphi \\
-\varphi^{*} & 0
\end{array}\right)+\left[\left(\begin{array}{cc}
0 & \varphi \\
-\varphi^{*} & 0
\end{array}\right),\left(\begin{array}{cc}
0 & \varphi \\
-\varphi^{*} & 0
\end{array}\right)\right] \\
& =F_{D}+\left(\begin{array}{cc}
-\varphi \wedge \varphi^{*} & 0 \\
0 & -\varphi^{*} \wedge \varphi
\end{array}\right)+\left(\begin{array}{cc}
0 & D_{2}^{*} \otimes D_{1}(\varphi) \\
D_{1}^{*} \otimes D_{2}(\varphi) & 0
\end{array}\right) .
\end{aligned}
$$

The harmonicity of $\varphi$ implies the vanishing of the last term; hence,

$$
\begin{aligned}
& \sqrt{-1} \Lambda F_{D^{\prime \prime}, H}= \\
& \quad=\left(\begin{array}{cc}
\sqrt{-1} \Lambda\left(F_{D_{1}^{\prime \prime}, H_{1}}-\varphi \wedge \varphi^{*}\right) & 0 \\
0 & \sqrt{-1} \Lambda\left(F_{D_{2}^{\prime \prime}, H_{2}}-\varphi^{*} \wedge \varphi\right)
\end{array}\right) .
\end{aligned}
$$

It follows that $\sqrt{-1} \Lambda F_{D^{\prime \prime}, H}=\tau_{1} \pi_{H}+\tau_{2} \pi_{H}^{\perp}$. By construction, we also have $\pi_{H}^{\perp} D^{\prime \prime}\left(\pi_{H}\right)=0$. Therefore, $\left(D^{\prime \prime}, H, \pi_{H}\right)$ satisfy (1.4).

Conversely, assume $\left(D^{\prime \prime}, H, \pi_{H}\right)$ satisfy (1.4). Then we set $E_{1}=\pi_{H}(E)$, $E_{2}=\pi_{H}^{\perp}(E)$. Let $D_{1}^{\prime \prime}, D_{2}^{\prime \prime}, H_{1}, H_{2}$ denote the induced holomorphic and hermitian structures from $D^{\prime \prime}, H$, and let $\varphi \in \Omega^{0,1}\left(\operatorname{Hom}\left(E_{2}, E_{1}\right)\right)$ denote the second fundamental form. From (1.6) and (1.7) we see that the equation $\sqrt{-1} \Lambda F_{D^{\prime \prime}, H}=\tau_{1} \pi_{H}+\tau_{2} \pi_{H}^{\frac{1}{H}}$ implies the first two equations in (1.1), as well as $D_{2}^{*} \otimes D_{1}(\varphi)=0$. The latter implies $\left(D_{2}^{\prime \prime}\right)^{*} \otimes D_{1}^{\prime \prime}(\varphi)=0$ and $\left(D_{2}^{\prime}\right)^{*} \otimes D_{1}^{\prime}(\varphi)=0$, which are equivalent to the harmonicity of $\varphi$. Hence, $\left(D_{1}^{\prime \prime}, D_{2}^{\prime \prime}, H_{1}, H_{2}, \varphi\right)$ satisfies (1.1).

We note the following relation between the parameters $\tau_{1}$ and $\tau_{2}$ : 
484 Georgios Daskalopoulos, Karen Uhlenbeck and Richard Wentworth

Lemma 1.8. Suppose equation (1.4) has a solution. Then under the normalization $\operatorname{Vol}(X)=2 \pi /(n-1) !, \tau_{1} R_{1}+\tau_{2}\left(R-R_{1}\right)=d_{1}+d_{2}$.

Proof. Take the trace of the first equation in (1.4) and integrate.

Notation 1.9. Set $R=R_{1}+R_{2}, d=d_{1}+d_{2}, \mu=d / R$, and $\tau=(-\mu+$ $\left.\tau_{2}\right) R / R_{1}$.

With the conventions above and in view of Lemma 1.8, equations (1.4) can be rewritten as

$$
\begin{cases}\sqrt{-1} \Lambda F_{D^{\prime \prime}, H} & =\left(\frac{R_{1}}{R} \tau+\mu\right) \mathbf{I}-\tau \pi_{H} \\ \pi_{H}^{\perp} D^{\prime \prime} \pi_{H} & =0 .\end{cases}
$$

Notice that when $\tau=0$, the first of these equations is just (1.3). Henceforth, we shall omit the notation $H$ when the metric is understood.

\subsection{Review of Kempf-Ness for Grassmannians.}

Given integers $R>R_{1}>0$, let

$$
\imath: \mathbb{G}\left(R_{1}, R\right) \hookrightarrow \mathbb{P}\left(\bigwedge^{R_{1}} \mathbb{C}^{R}\right) \simeq \mathbb{P}^{\left(\begin{array}{l}
R \\
R_{1}
\end{array}\right)-1}=\mathbb{P}
$$

denote the Plücker embedding of the Grassmannian of $R_{1}$ dimensional subspaces of $\mathbb{C}^{R}$ into projective space (cf. [GH],p. 193). This endows $\mathbb{G}\left(R_{1}, R\right)$ with the structure of a Kähler manifold. The image of the Grassmannian is invariant with respect to the action of $S L(R)$ on $\mathbb{P}$. Let $\rho: S L(R) \rightarrow P G L\left(\left(\begin{array}{c}R \\ R_{1}\end{array}\right)\right)$ denote the induced representation.

Let $\gamma$ be the pullback of the anti-tautological line bundle on projective space via the Plücker embedding. The action of $S L(R)$ via $\rho$ lifts to $\gamma$, and we may assume that $\gamma$ has an invariant hermitian metric and connection. Since everything is pulled back from $\mathbb{P}$, the curvature of $\gamma$ is the Kähler form on $\mathbb{G}\left(R_{1}, R\right)$. It follows that the unique Ad-equivariant moment map $\mu$ for the action of $S U(R)$ on $\mathbb{G}\left(R_{1}, R\right)$ is given by the composition

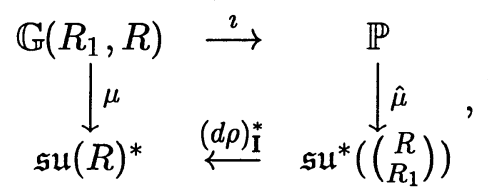

where $\hat{\mu}$ is the moment map for the action of $P G L\left(\left(\begin{array}{l}R \\ R_{1}\end{array}\right)\right)$ on $\mathbb{P}$. 
Lemma 1.11. Given $V \in \mathbb{G}\left(R_{1}, R\right)$, let $\pi^{V}: \mathbb{C}^{R} \rightarrow \mathbb{C}^{R}$ denote the projection onto $V$, i.e. $\pi^{V}\left(\mathbb{C}^{R}\right)=V, \pi^{V}=\left(\pi^{V}\right)^{*}=\left(\pi^{V}\right)^{2}$. Then for $\xi \in \mathfrak{s u}(R)$, $\mu(V)(\xi)=-\sqrt{-1} \operatorname{tr}\left(\pi^{V} \cdot \xi\right)$.

Proof. Fix an orthonormal basis $e_{1}, \ldots, e_{R}$ for $\mathbb{C}^{R}$. Since $\pi^{g V}=g \pi^{V} g^{-1}$ for $g \in S U(R)$, the map

$$
\mathbb{G}\left(R_{1}, R\right) \longrightarrow \mathfrak{s u}(R): V \mapsto \sqrt{-1} \pi V
$$

is Ad-equivariant. Then since $S U(R)$ acts transitively on $\mathbb{G}\left(R_{1}, R\right)$, it suffices to check the result when $V=\operatorname{span}\left\{e_{1}, \ldots, e_{R_{1}}\right\}$. Let

$$
\mathcal{I}=\left\{\left(i_{1}, \ldots, i_{R_{1}}\right): i_{j} \in\{1, \ldots, R\}, i_{1}<\cdots<i_{R_{1}}\right\}
$$

Then $\left\{w_{I}=e_{i_{1}} \wedge \cdots \wedge e_{i_{R_{1}}}: I \in \mathcal{I}\right\}$ forms a basis for $\bigwedge^{R_{1}} \mathbb{C}^{R}$. With respect to this basis,

$$
d \rho_{\mathbf{I}}(\xi)_{I J}=\sum_{l=1}^{R_{1}} \sum_{\sigma} \delta_{i_{1} j_{\sigma(1)}} \cdots \xi_{i_{l} j_{\sigma(l)}} \cdots \delta_{i_{R_{1}} j_{\sigma\left(R_{1}\right)}},
$$

for $\xi_{i j}$ the matrix entries of $\xi \in \mathfrak{s u}(R)$ with respect to $\left\{e_{j}\right\}$, and where the second sum is over all permutations $\sigma$. The moment map for the action on projective space may be found in [Ki]: Up to a normalization of the Fubini-Study metric, we have

$$
\hat{\mu}(\imath V)\left(d \rho_{\mathbf{I}}(\xi)\right)=-\frac{\sqrt{-1}}{\|\widetilde{\imath V}\|^{2}} \sum_{I J}\left(d \rho_{\mathbf{I}}(\xi)\right)_{I J}(\widetilde{\imath V})_{I}(\widetilde{\imath V})_{J}
$$

where the tilde denotes any lift from the projective space to $\bigwedge^{R_{1}} \mathbb{C}^{R}$. For our choice of $V$ we may take

$$
(\widetilde{\imath V})_{I}= \begin{cases}1 & \text { if } I=I_{0}=\left\{1, \ldots, R_{1}\right\} \\ 0 & \text { otherwise }\end{cases}
$$

Hence,

$$
\hat{\mu}(\imath V)\left(d \rho_{\mathbf{I}}(\xi)\right)=-\sqrt{-1} d \rho_{\mathbf{I}}(\xi)_{I_{0} I_{0}}=-\sqrt{-1} \sum_{l=1}^{R_{1}} \xi_{l l}=-\sqrt{-1} \operatorname{tr}\left(\pi^{V} \cdot \xi\right)
$$

Let $\|\cdot\|_{\gamma}$ denote the hermitian metric on $\gamma$. Given $g \in S L(R)$ and $V \in \mathbb{G}\left(R_{1}, R\right)$, let

$$
\nu(V, g)=\log \frac{\|g \hat{V}\|_{\gamma}^{2}}{\|\hat{V}\|_{\gamma}^{2}}
$$


486 Georgios Daskalopoulos, Karen Uhlenbeck and Richard Wentworth

where $\hat{V}$ is any non-zero lift of $V$ to the fiber of $\gamma$ over $V$. By the Kempf-Ness theorem, if $s \in \sqrt{-1} \mathfrak{s u}(R)$, and $g_{t}=e^{t s}$, then

$$
\begin{aligned}
& \frac{d \nu}{d t}=-4 \pi \mu\left(g_{t} V\right)(-\sqrt{-1} s)=4 \pi \operatorname{tr}\left(\pi^{\left(g_{t} V\right)} \cdot s\right) \\
& \frac{d^{2} \nu}{d t^{2}} \geq 0 .
\end{aligned}
$$

(See [KN]; also, regarding the normalizations in the first equation of (1.12), we refer to [GS, 5.5]). The next result will be used in $\S 3$ :

Lemma 1.13. With the notation as in Lemma 1.11, and for $s \in$ $\sqrt{-1} \mathfrak{s u}(R)$,

$$
\nu\left(V, e^{s}\right) \geq 4 \pi \operatorname{tr}\left(s \pi^{V}\right) .
$$

Proof. Set $\lambda(t)=\nu\left(V, e^{t s}\right)+4 \pi(1-t) \operatorname{tr} s \pi^{e^{t s} V}$. By (1.11) and (1.12),

$$
\begin{aligned}
\lambda^{\prime}(t) & =4 \pi \operatorname{tr} s \pi^{e^{t s} V}-4 \pi \operatorname{tr} s \pi^{e^{t s} V}+4 \pi(1-t) \operatorname{tr} s \frac{d}{d t} \pi^{e^{t s} V} \\
& =(1-t) \frac{d^{2} \nu}{d t^{2}} \geq 0, \quad 0 \leq t \leq 1 .
\end{aligned}
$$

Thus, $\nu\left(V, e^{s}\right)=\lambda(1) \geq \lambda(0)=4 \pi \operatorname{tr}\left(s \pi^{V}\right)$.

We end this subsection with the following:

Lemma 1.14. Let $\pi_{K}^{V}$ denote the projection operator onto $V$ with respect to the metric $K$, and let $s \in \sqrt{-1} \mathfrak{s u}(R)$. Then $e^{-s} \pi_{K}^{e^{s} V} e^{s}=\pi_{e^{2 s} K}^{V}$.

Proof. Both $e^{-s} \pi_{K}^{e^{s} V} e^{s}$ and $\pi_{e^{2 s} K}^{V}$ project $\mathbb{C}^{R}$ onto $V$ and are idempotent; hence it suffices to check that

$$
\left(e^{-s} \pi_{K}^{e^{s} V} e^{s}\right)^{*} e^{2 s} K=e^{-s} \pi_{K}^{e^{s} V} e^{s}
$$

Indeed,

$$
\begin{aligned}
\left(e^{-s} \pi_{K}^{e^{s} V} e^{s}\right)^{*} e^{2 s_{K}} & =e^{-2 s}\left(e^{-s} \pi_{K}^{e^{s} V} e^{s}\right)^{*_{K}} e^{2 s} \\
& =e^{-2 s}\left(e^{s} \pi_{K}^{e^{s} V} e^{-s}\right) e^{2 s} \\
& =e^{-s} \pi_{K}^{e^{s} V} e^{s}
\end{aligned}
$$

\subsection{Moment map interpretation.}

Using Lemma 1.11, we reinterpret equations (1.10) in the setting of infinite dimensional sympectic geometry. Let $E$ be a vector of rank $R=R_{1}+R_{2}$ 
with a fixed background hermitian metric $K, P$ the frame bundle associated to $E$, and $\mathbb{G}\left(R_{1}, R\right)$ the Grassmannian as in the previous section. Let

$$
\mathbb{G}\left(R_{1}, E\right)=P \times_{U(R)} \mathbb{G}\left(R_{1}, R\right)
$$

be the Grassmann bundle associated to $P$, and let $\Omega^{0}\left(\mathbb{G}\left(R_{1}, E\right)\right)$ be the space of global smooth sections. It is well-known (cf. [DU]) that $\Omega^{0}\left(\mathbb{G}\left(R_{1}, E\right)\right)$ is a smooth manifold with tangent space $T_{\pi} \Omega^{0}\left(\mathbb{G}\left(R_{1}, E\right)\right)=\Omega^{0}\left(\operatorname{Hom}\left(\pi, \pi^{\perp}\right)\right)$. Furthermore, the inclusion $\Omega^{0}\left(\operatorname{Hom}\left(\pi, \pi^{\perp}\right)\right)$ in $\Omega^{0}(\operatorname{End} E)$ with the $L^{2}$ metric endows $\Omega^{0}\left(G\left(R_{1}, E\right)\right)$ with the structure of a Kähler manifold. Let $\omega_{\Omega}$ denote its Kähler form. Clearly, for $\xi, \eta \in \Omega^{0}\left(\mathbb{G}\left(R_{1}, E\right)\right)$,

$$
\omega_{\Omega}(\xi, \eta)=\frac{1}{2 \sqrt{-1}} \int_{X}\left(\langle\xi, \eta\rangle_{K}-\langle\eta, \xi\rangle_{K}\right) \omega^{n} .
$$

The real gauge group $\mathfrak{G}$ of $E$ acts on $\mathbb{G}\left(R_{1}, E\right)$ preserving the symplectic form. According to Lemma 1.11, an associated Ad-equivariant moment map $\mu_{\Omega}$ is given by

$$
\mu_{\Omega}(\pi) \cdot \xi=-\sqrt{-1} \int_{X} \operatorname{tr}(\pi \xi) \omega^{n} .
$$

Next, recall that the space $\mathcal{A}$ of hermitian connections (or equivalently, $\bar{\partial}$ operators) on $E$ is an affine space modelled on $\Omega^{1}(\operatorname{ad} E)$. The identification $\Omega^{1}(\operatorname{ad} E) \simeq \Omega^{0,1}($ End $E)$ endows $\mathcal{A}$ with the structure of a Kähler manifold. The symplectic form is given by (cf. [Ko], VII, $\S 6$ )

$$
\begin{aligned}
\omega_{\mathcal{A}}(\alpha, \beta) & =\frac{1}{2 \sqrt{-1}}\left(\langle\alpha, \beta\rangle_{L^{2}}-\langle\beta, \alpha\rangle_{L^{2}}\right) \\
& =\frac{n}{2} \int_{X} \operatorname{tr} \alpha \wedge \beta \wedge \omega^{n-1}, \quad \alpha, \beta \in \Omega^{1}(\operatorname{ad} E),
\end{aligned}
$$

and a moment map for the action of $\mathfrak{G}$ on $\mathcal{A}$ is given by

$$
\mu_{\mathcal{A}}(D) \cdot \xi=\frac{1}{2} \int_{X} \operatorname{tr}\left(\Lambda F_{D} \xi\right) \omega^{n} .
$$

Now consider the product $\mathcal{A} \times \Omega^{0}\left(\mathbb{G}\left(R_{1}, E\right)\right)$ with Kähler form

$$
\omega_{\tau}=2 \omega_{\mathcal{A}}+n ! \tau \omega_{\Omega} .
$$

It follows from (1.15) and (1.16) that

Proposition 1.18. The moment map for the action of $\mathfrak{G}$ on $\mathcal{A} \times$ $\Omega^{0}\left(\mathbb{G}\left(R_{1}, E\right)\right)$ with respect to $\omega_{\tau}$ is given by

$$
\mu_{\tau}(D, \pi) \cdot \xi=\int_{X} \operatorname{tr}\left\{\left(\Lambda F_{D}-\tau \sqrt{-1} \pi\right) \xi\right\} \omega^{n} .
$$


488 Georgios Daskalopoulos, Karen Uhlenbeck and Richard Wentworth

Definition 1.20. Let $\mathcal{H} \subset \mathcal{A} \times \Omega^{0}\left(\mathbb{G}\left(R_{1}, E\right)\right)$ denote the subspace consisting of pairs $(D, \pi)$ such that $\left(D^{\prime \prime}\right)^{2}=0$ and $\pi^{\perp} D^{\prime \prime}(\pi)=0$.

In general, $\mathcal{H}$ will have singularities, a situation which will be analyzed in $\S 4$. For the purpose of this section, we state the following evident

Proposition 1.21. Let $a=-\sqrt{-1}\left(\tau R_{1} / R+\mu\right)$ I. Then the solutions to (1.10) correspond to $\mu_{\tau}^{-1}(a) \cap \mathcal{H}$.

\section{Stable Extensions.}

\subsection{Mumford and Gieseker stability.}

Definition 2.1. An extension pair $\left(E_{1}, E\right)$ consists of a coherent analytic sheaf $E$ and a subsheaf $E_{1}$. A subpair $\left(E_{1}^{\prime}, E^{\prime}\right)$ of $\left(E_{1}, E\right)$ is an extension pair such that $E^{\prime}$ is a subsheaf of $E$, and $E_{1}^{\prime}$ is the subsheaf of $E^{\prime}$ induced from $E_{1}$. A subpair is proper if $E^{\prime}$ is neither zero nor $E$. A pair is called torsion-free (resp. locally-free) if $E$ is a torsion-free (resp. locally-free) sheaf. It is called saturated if the subsheaf $E_{1}$ is saturated, i.e. the quotient sheaf $E / E_{1}$ is torsion-free, and it is called smooth if $E$ is locally-free and $E_{1}$ is a subbundle.

Recall that given a coherent analytic sheaf $F$, we can define the $\omega$ degree as for bundles. In case $\operatorname{rk} F \neq 0$, we also define the $\omega$-slope $\mu(F)=$ $\operatorname{deg}(F) / \operatorname{rk}(F)$.

Definition 2.2. Let $\left(E_{1}, E\right)$ be an extension pair with $\operatorname{rk} E \neq 0$ and $\tau \in \mathbb{R}$. We define

$$
\mu_{\tau}^{S}\left(E_{1}, E\right)=\mu(E)+\tau \frac{\mathrm{rk} E_{1}}{\mathrm{rk} E} .
$$

A torsion-free extension pair $\left(E_{1}, E\right)$ is called $\tau$-slope stable (resp. $\tau$-slope semistable $)$ if for any proper subpair $\left(E_{1}^{\prime}, E^{\prime}\right)$ of $\left(E_{1}, E\right)$ we have

$$
\left.\mu_{\tau}^{S}\left(E_{1}^{\prime}, E^{\prime}\right)<\mu_{\tau}^{S}\left(E_{1}, E\right) \quad \text { (resp. } \leq\right) .
$$

A pair is called $\tau$-slope polystable if we have a holomorphic splitting

$$
\left(E_{1}, E\right)=\bigoplus_{k=1}^{m}\left(E_{1}^{(k)}, E^{(k)}\right),
$$

where $\left(E_{1}^{(k)}, E^{(k)}\right)$ is $\tau$-slope stable for $k=1, \ldots, m$. 
From the point of view of algebraic geometry, it is more natural to consider the dual situation. Thus

Definition 2.3. A quotient pair $q: E \rightarrow F \rightarrow 0$ consists of a coherent sheaf $E$ and a quotient $F$. A subquotient $q: E^{\prime} \rightarrow F^{\prime}$ is a subsheaf $E^{\prime}$ and its image $F^{\prime} \subset F$ by $q$. A subquotient is proper if $E^{\prime}$ is neither zero nor $E$. We shall call a quotient torsion-free if $E$ is a torsion-free sheaf.

For a quotient $q: E \rightarrow F \rightarrow 0$, where $\operatorname{rk} E \neq 0$, we define the $\tau$-slope:

$$
\mu_{\tau}^{Q}(E, F)=\mu(E)-\tau \frac{\operatorname{rk} F}{\operatorname{rk} E} .
$$

Definition 2.4. We say a torsion-free quotient $q: E \rightarrow F \rightarrow 0$ is $\tau$-slope semistable (resp. $\tau$-slope stable) if for all proper subsheaves $0 \neq E^{\prime} \subset E$ with image $F^{\prime} \subset F$ under $q$, we have

$$
\mu_{\tau}^{Q}\left(E^{\prime}, F^{\prime}\right) \leq \mu_{\tau}^{Q}(E, F) \quad(\text { resp. } \quad<)
$$

The following is clear from the definitions:

Proposition 2.5. If $q: E \rightarrow F \rightarrow 0$ is a $\tau$-(semi)stable quotient pair, then $\left(E_{1}, E\right)$, where $E_{1}=\operatorname{ker} q$ is a $\tau$-(semi)stable extension pair. Conversely, if $\left(E_{1}, E\right)$ is a $\tau$-(semi)stable extension pair, then $q: E \rightarrow F \rightarrow 0$, where $F=E / E_{1}$ and $q$ is the projection, is a $\tau$-(semi)stable quotient pair.

We also introduce the notion of Gieseker stability in case the Kähler manifold $X$ is actually projective. This is a refinement of slope semistability, even in the case of Riemann surfaces. So suppose $X$ is now a projective algebraic manifold with an ample line bundle $A$. We shall assume the Kähler form $\omega$ represents $c_{1}(A)$. Recall that the Hilbert polynomial of a coherent sheaf $E$ on $X$ with respect to the ample line bundle $A$ is given by $\chi\left(E \otimes A^{k}\right)$, which is $h^{0}\left(E \otimes A^{k}\right)$ for $k$ large. When $\operatorname{rk} E \neq 0$, we shall often use the normalized polynomial $p(E, k)=\chi\left(E \otimes A^{k}\right) / \mathrm{rk} E$.

Definition 2.6. Let $q: E \rightarrow F \rightarrow 0$ be a torsion-free quotient pair. If $\operatorname{rk} F=0$, then we shall call the pair $\tau$-Gieseker semistable (resp. stable) if $E$ is Gieseker semistable (resp. stable) in the usual sense. If $\operatorname{rk} F \neq 0$, then the pair is $\tau$-Gieseker semistable (resp. stable) if for all proper subsheaves $E^{\prime} \subset E$ the following conditions hold:

(i) $\mu_{\tau}^{Q}\left(E^{\prime}, F^{\prime}\right) \leq \mu_{\tau}^{Q}(E, F)$; 
490 Georgios Daskalopoulos, Karen Uhlenbeck and Richard Wentworth

(ii) If $\mu_{\tau}^{Q}\left(E^{\prime}, F^{\prime}\right)=\mu_{\tau}^{Q}(E, F)$, then $p\left(E^{\prime}, k\right) \leq p(E, k)$ for $k>>0$;

(iii) If $\mu_{\tau}^{Q}\left(E^{\prime}, F^{\prime}\right)=\mu_{\tau}^{Q}(E, F)$, and $p\left(E^{\prime}, k\right)=p(E, k)$ for all $k$, then $p\left(F^{\prime}, k\right) \geq p(F, k)($ resp. $>$ ) for $k>>0$.

The following is straightforward:

Lemma 2.7. In Definitions 2.2, 2.4, and 2.6, it suffices to consider only those $E^{\prime}$ with torsion-free quotients $E / E^{\prime}$.

We also note that as in the case of semistability for sheaves, we have

$$
\begin{gathered}
\tau \text {-slope stable } \Longrightarrow \tau \text {-Gieseker stable } \Longrightarrow \\
\tau \text {-Gieseker semistable } \Longrightarrow \tau \text {-slope semistable }
\end{gathered}
$$

To make contact with the existence of special metrics, we would like to associate to an arbitrary quotient pair $E \rightarrow F \rightarrow 0$, where $E$ is locallyfree, a locally free, saturated extension pair $\left(E_{1}, E\right)$. However, we shall see that the stability condition alone may not ensure that $F$ be torsion-free. Nevertheless, we do have the following:

Proposition 2.8. Suppose $E \rightarrow F \rightarrow 0$ is a torsion-free quotient pair, and let $T$ denote the torsion subsheaf of $F$. Then $E \rightarrow F \rightarrow 0$ is $\tau$ Gieseker semistable (resp. stable) if and only if the quotient $E \rightarrow F / T \rightarrow$ 0 is $\tau$-Gieseker semistable (resp. stable). The same is true for slope (semi)stability.

Proof. Consider conditions (i) and (ii) in Definition 2.6. These hold for $E \rightarrow F \rightarrow 0$ if and only if they hold for $E \rightarrow F / T \rightarrow 0$, since only the ranks of $F$ and $F / T$ are involved, and they are equal. This proves the last remark. For the other conditions, we need the following

Lemma 2.9. Suppose $E \rightarrow F \rightarrow 0$ is a quotient for which conditions (i) and (ii) of Definition 2.6 hold, and suppose that $E^{\prime} \subset E$ is a proper subsheaf giving equality in (ii). Then the torsion $T$ of $F$ is contained in the image $F^{\prime}$ of $E^{\prime}$.

Proof. Let $T\left(F / F^{\prime}\right)$ denote the torsion subsheaf of $F / F^{\prime}$. Let $\widehat{F}^{\prime} \subset F$ be the kernel of

$$
F \longrightarrow\left(F / F^{\prime}\right) / T\left(F / F^{\prime}\right) \longrightarrow 0
$$


and let $E^{\prime \prime} \subset E$ be the kernel of $E \rightarrow F / \widehat{F}^{\prime} \rightarrow 0$. Then $E^{\prime} \subset E^{\prime \prime}$, and the quotient $E^{\prime \prime} / E^{\prime}$ is isomorphic to the torsion sheaf $\widehat{F}^{\prime} / F^{\prime}$. But by assumption, we must have $p\left(E^{\prime \prime}, n\right)=p(E, n)$ for all $n$, so the Hilbert polynomial of $\widehat{F}^{\prime} / F$ is zero. Hence, $\widehat{F}^{\prime}=F^{\prime}$, and $T\left(F / F^{\prime}\right)=0$. This proves the claim.

Continuing with the proof of Proposition 2.8, by the previous remark we may assume $E \rightarrow F \rightarrow 0$ is as in Lemma 2.9. If $E^{\prime} \subset E$ is a subsheaf for which equality holds in Definition 2.6 (i) and (ii), then by the lemma, $p(F, k) \leq p\left(F^{\prime}, k\right)$ (resp. $<$ ) for large $k$ if and only if $p(F / T, k) \leq p\left(F^{\prime} / T, k\right)$ (resp. $<$ ) for large $k$. This completes the proof.

\subsection{Simplicity.}

A homomorphism of quotient pairs $q: E \rightarrow F \rightarrow 0$ and $q^{\prime}: E^{\prime} \rightarrow F^{\prime} \rightarrow 0$ is a pair of sheaf homomorphisms $\phi: E \rightarrow E^{\prime}$ and $\psi: F \rightarrow F^{\prime}$ making the following diagram commute:

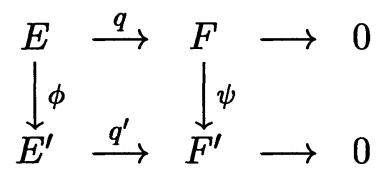

We say $q$ is isomorphic to $q^{\prime}$ if both $\phi$ and $\psi$ are isomorphisms. Clearly, the condition on a homomorphism $\phi: E \rightarrow E^{\prime}$ to give rise to a quotient homomorphism is $\phi(\operatorname{ker} q) \subset \operatorname{ker} q^{\prime}$. Notice that $\psi$ is then completely determined by $\phi$.

Similarly, for extension pairs $\left(E_{1}, E\right)$ and $\left(E_{1}^{\prime}, E^{\prime}\right)$, a homomorphism of pairs is a homomorphism $\phi: E \rightarrow E^{\prime}$ satisfying $\phi\left(E_{1}\right) \subset E_{1}^{\prime}$.

Definition 2.10. An extension pair $\left(E_{1}, E\right)$ or a quotient pair $E \rightarrow F \rightarrow 0$ is called simple if the only endomorphisms are scalar multiples of the identity.

Proposition 2.11. For any $\tau \geq 0$, any $\tau$-Gieseker stable quotient pair is simple.

Proof. Let $q: E \rightarrow F \rightarrow 0$ be $\tau$-Gieseker stable suppose that $\phi: E \rightarrow E$ is an endomorphism with $\phi(\operatorname{ker} q) \subset \operatorname{ker} q$. As in the case of semistable sheaves (cf. [Nw]) we may assume without loss of generality that $E^{\prime}=\operatorname{ker} \phi$ is a proper subsheaf of $E$. If $E^{\prime \prime}$ denotes the image of $\phi$, we have the induced 
492 Georgios Daskalopoulos, Karen Uhlenbeck and Richard Wentworth sequence:

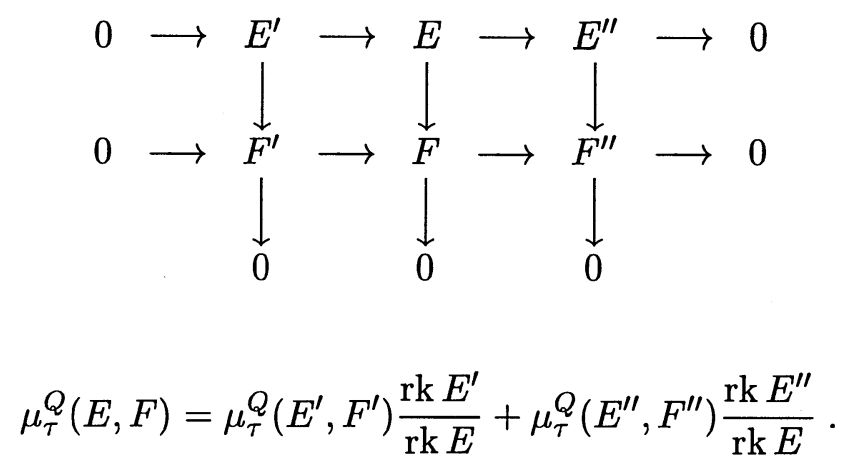

For $k$ large, we also have

$$
\begin{aligned}
& p(E, k)=p\left(E^{\prime}, k\right) \frac{\mathrm{rk} E^{\prime}}{\operatorname{rk} E^{\prime}}+p\left(E^{\prime \prime}, k\right) \frac{\operatorname{rk} E^{\prime \prime}}{\mathrm{rk} E^{\prime \prime}} \\
& p(F, k)=p\left(F^{\prime}, k\right) \frac{\mathrm{rk} F^{\prime}}{\operatorname{rk} F}+p\left(F^{\prime \prime}, k\right) \frac{\mathrm{rk} F^{\prime \prime}}{\operatorname{rk} F} .
\end{aligned}
$$

But stability contradicts at least one of the three equalities above.

By Proposition 2.5, or a similar direct argument, we also have Proposition 2.12. Every $\tau$-slope stable extension pair is simple.

\subsection{Jordan-Hölder filtrations.}

For the purpose of constructing a Hausdorff moduli space, we shall have to deal with Seshadri equivalence.

Proposition 2.13. Let $E \rightarrow F \rightarrow 0$ be a $\tau$-Gieseker semistable torsion-free quotient pair. Then there is a filtration

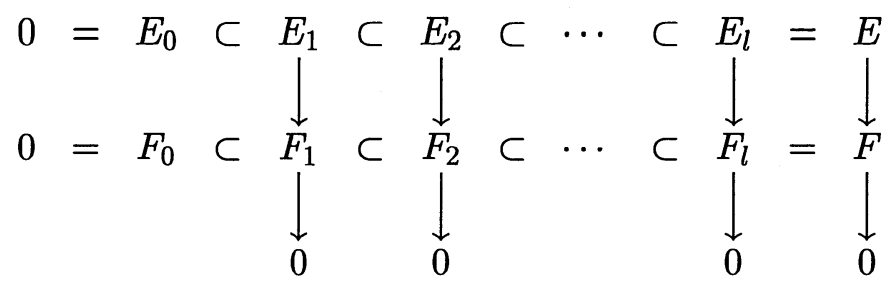

such that $E_{i-1}$ is saturated in $E_{i}$, and the induced quotients

$$
E_{i} / E_{i-1} \longrightarrow F_{i} / F_{i-1} \longrightarrow 0
$$


are $\tau$-Gieseker stable. Moreover, the sum of quotients

$$
\operatorname{gr}(E, F)=\left(E_{1}, F_{1}\right) \oplus \cdots \oplus\left(E_{i} / E_{i-1}, F_{i} / F_{i-1}\right) \oplus \cdots \oplus\left(E / E_{l-1}, F / F_{l-1}\right)
$$

is unique up to isomorphism.

Proof. By induction on the rank of $E$. Suppose $E \rightarrow F \rightarrow 0$ is as in the statement of the proposition. By definition, there exists a saturated $E^{\prime} \subset E$ and induced quotient $E^{\prime} \rightarrow F^{\prime} \rightarrow 0$ such that

(i) $\mu_{\tau}^{Q}\left(E^{\prime}, F^{\prime}\right)=\mu_{\tau}^{Q}(E, F)$;

(ii) $p\left(E^{\prime}, k\right)=p(E, k)$ for all $k$;

(iii) $p\left(F^{\prime}, k\right)=p(F, k)$ for all $k$, and $E^{\prime}$ is maximal with respect to all such subsheaves of $E$.

Clearly, $E^{\prime} \rightarrow F^{\prime} \rightarrow 0$ is itself $\tau$-semistable. Consider $E^{\prime \prime} \rightarrow F^{\prime \prime} \rightarrow 0$, where $E^{\prime \prime}=E / E^{\prime}$ and $F^{\prime \prime}=F / F^{\prime}$. Then a simple computation shows (i), (ii), and (iii) above hold with $E^{\prime}$ and $F^{\prime}$ replaced by $E^{\prime \prime}$ and $F^{\prime \prime}$. By induction, we need only show that $E^{\prime \prime} \rightarrow F^{\prime \prime} \rightarrow 0$ is $\tau$-Gieseker semistable. Suppose to the contrary. Thus, let $\hat{E} \subset E^{\prime \prime}$ be a proper subsheaf with torsion-free quotient, and let $\hat{E} \rightarrow \hat{F} \rightarrow 0$ be the induced quotient pair. We first assume $\mu_{\tau}^{Q}(\hat{E}, \hat{F})>\mu_{\tau}^{Q}\left(E^{\prime \prime}, F^{\prime \prime}\right)=\mu_{\tau}^{Q}(E, F)$. Let $\hat{E}^{\prime}$ be the preimage of $\hat{E}$, i.e. the kernel of the induced map $E \rightarrow E^{\prime \prime} / \hat{E} \rightarrow 0$. Then

$$
\begin{gathered}
\mu_{\tau}^{Q}(E, F)=\mu_{\tau}^{Q}\left(\hat{E}^{\prime}, \hat{F}^{\prime}\right) \frac{\mathrm{rk} \hat{E}^{\prime}}{\mathrm{rk} E}+\mu_{\tau}^{Q}\left(E^{\prime \prime} / \hat{E}, F^{\prime \prime} / \hat{F}\right) \frac{\mathrm{rk} E^{\prime \prime} / \hat{E}}{\mathrm{rk} E} . \\
\mu_{\tau}^{Q}\left(E^{\prime \prime} / \hat{E}, F^{\prime \prime} / \hat{F}\right)<\mu_{\tau}^{Q}\left(E^{\prime \prime}, F^{\prime}\right)=\mu_{\tau}^{Q}(E, F) .
\end{gathered}
$$

But then

$$
\begin{aligned}
& \mu_{\tau}^{Q}(E, F)\left(\frac{\mathrm{rk} E-\mathrm{rk} E^{\prime \prime} \mid \hat{E}}{\mathrm{rk} E}\right)<\mu_{\tau}^{Q}\left(\hat{E}^{\prime}, \hat{F}^{\prime}\right) \frac{\mathrm{rk} \hat{E}^{\prime}}{\mathrm{rk} E} \\
& \Longrightarrow \quad \mu_{\tau}^{Q}(E, F) \quad<\mu_{\tau}^{Q}\left(\hat{E}^{\prime}, \hat{F}^{\prime}\right),
\end{aligned}
$$

contradicting semistability. The other conditions in the definition of $\tau$ Gieseker semistability are easy to check.

\section{The Hitchin-Kobayashi Correspondence.}

The main goal of this section is to prove the correspondence between locally-free, saturated $\tau$-stable extension pairs $\left(E_{1}, E\right)$ and solutions to equations (1.10). To fix notation, let $p \geq 2 n, n=\operatorname{dim}_{\mathbb{C}} X$ and let $M e t_{2}^{p}$ denote 
494 Georgios Daskalopoulos, Karen Uhlenbeck and Richard Wentworth

the space of hermitian metrics on $E$ of class $L_{2}^{p}$. Given $\chi$ a smooth hermitian metric on $\bigwedge^{R} E=\operatorname{det} E$, we let $\operatorname{Met}_{2}^{p}(\chi)$ denote the space of hermitian metrics in $M e t_{2}^{p}$ of determinant $\chi$. We now formulate our main theorem:

Theorem 3.1. Suppose that $\left(E_{1}, E\right)$ is a locally-free, saturated, $\tau$-stable extension pair with respect to the holomorphic structure $D^{\prime \prime}$ on $E$. Then there is a unique hermitian metric $H \in M_{2}^{p} t_{2}^{p}$ such that equations (1.10) are satisfied. Conversely, if $\left(D^{\prime \prime}, H, \pi_{H}\right)$ is a solution to equations (1.10), then $\left(E_{1}=\pi_{H}(E), E\right)$ is a $\tau$-polystable pair.

\subsection{Necessary conditions.}

We begin with the easy direction:

Proposition 3.2. (Necessary conditions) Suppose that $\left(D^{\prime \prime}, H, \pi_{H}\right)$ is a solution to equations (1.10) with $H \in M e t_{2}^{p}$. Then $\left(E_{1}=\pi_{H}(E), E\right)$ is a polystable pair.

Proof. Let $\left(E_{1}^{\prime}, E^{\prime}\right)$ be a subpair of $\left(E_{1}, E\right)$. Let $\pi_{H}^{\prime}$ denote the weak projection corresponding to $E^{\prime}$ (and $H$ ). We may assume that $\pi_{H}^{\prime}$ defines a subbundle of $E$ outside a set $\Sigma$ of complex codimension $\geq 2$. Let

$$
\Theta=\left(\begin{array}{cc}
\vartheta^{\prime} & A \\
-A^{*} & \vartheta^{\perp}
\end{array}\right)
$$

denote a local frame in $X \backslash \Sigma$ of the hermitian connection on $E$ corresponding to $\left(D^{\prime \prime}, H\right)$. In the above notation, $\vartheta^{\prime}$ is the connection form on $E^{\prime}$ and $\vartheta^{\perp}$ that on $\left(E^{\prime}\right)^{\perp}$. By the assumption that $\left(D^{\prime \prime}, H, \pi_{H}\right)$ satisfies (1.10) we have

$$
\begin{aligned}
& \left(\begin{array}{cc}
\sqrt{-1} \Lambda F^{\prime}+\sqrt{-1} \Lambda\left(A \wedge A^{*}\right) & * \\
* & \sqrt{-1} \Lambda F^{\perp}+\sqrt{-1} \Lambda\left(A^{*} \wedge A\right)
\end{array}\right) \\
& =\left(\mu-\frac{R-R_{1}}{R} \tau\right) \pi_{H}+\left(\mu+\frac{R_{1}}{R} \tau\right) \pi_{H}^{\perp},
\end{aligned}
$$

hence,

$$
\begin{aligned}
\sqrt{-1} \pi_{H}^{\prime} \Lambda F \pi_{H}^{\prime}= & \sqrt{-1} \Lambda F^{\prime}+\sqrt{-1} \Lambda\left(A \wedge A^{*}\right) \\
& =\left(\mu-\frac{R-R_{1}}{R} \tau\right) \pi_{H}^{\prime} \pi_{H} \pi_{H}^{\prime}+\left(\mu+\frac{R_{1}}{R} \tau\right) \pi_{H}^{\prime} \pi_{H}^{\perp} \pi_{H}^{\prime}
\end{aligned}
$$




$$
\begin{aligned}
& \sqrt{-1}\left(\pi_{H}^{\prime}\right)^{\perp} \Lambda F\left(\pi_{H}^{\prime}\right)^{\perp}=\sqrt{-1} \Lambda F^{\perp}+\sqrt{-1} \Lambda\left(A^{*} \wedge A\right) \\
& \quad=\left(\mu-\frac{R-R_{1}}{R} \tau\right)\left(\pi_{H}^{\prime}\right)^{\perp} \pi_{H}\left(\pi_{H}^{\prime}\right)^{\perp}+\left(\mu+\frac{R_{1}}{R} \tau\right)\left(\pi_{H}^{\prime}\right)^{\perp} \pi_{H}^{\perp}\left(\pi_{H}^{\prime}\right)^{\perp}
\end{aligned}
$$

Since $\Lambda F \in L^{p}$ and $\pi_{H}^{\prime} \in L^{\infty}$, we may integrate the first equation over $X$ (recall the normalization $\operatorname{Vol}(X)=2 \pi /(n-1)$ !) to obtain

$$
\begin{aligned}
\operatorname{deg} E^{\prime}+C_{A}=\left(\mu-\frac{R-R_{1}}{R} \tau\right) & \operatorname{rk}\left(\pi_{H}^{\prime} \cap \pi_{H}\right) \\
& +\left(\mu+\frac{R_{1}}{R} \tau\right)\left(\operatorname{rk} \pi_{H}^{\prime}-\operatorname{rk}\left(\pi_{H}^{\prime} \cap \pi_{H}\right)\right)
\end{aligned}
$$

where

$$
0 \leq C_{A}=\frac{\sqrt{-1}}{2 \pi n} \int_{X} \operatorname{tr} \Lambda\left(A \wedge A^{*}\right) \omega^{n} .
$$

By setting $R^{\prime}=\mathrm{rk} E^{\prime}, R_{1}^{\prime}=\mathrm{rk} E_{1}^{\prime}$, we obtain

$$
\operatorname{deg} E^{\prime} \leq\left(\mu-\frac{R-R_{1}}{R} \tau\right) R_{1}^{\prime}+\left(\mu+\frac{R_{1}}{R} \tau\right)\left(R^{\prime}-R_{1}^{\prime}\right)
$$

which is equivalent to $\mu_{\tau}^{S}\left(E_{1}^{\prime}, E^{\prime}\right) \leq \mu_{\tau}^{S}\left(E_{1}, E\right)$. If we have equality, then $C_{A}=0$. Hence, $A=0$, and by an argument as in [Br, 2.6], $E^{\prime}$ is locally-free and there is a splitting $E=E^{\prime} \oplus E^{\perp}$. But then the equations (1.10) also split, so by induction on the rank, $\left(E_{1}, E\right)$ is polystable.

Before going on to prove the converse statement, it will be useful to reformulate our equations after we fix the determinant of $E$. More precisely, given $A$ an endomorphism of $E$, let

$$
\hat{A}=A-\frac{1}{R} \operatorname{tr} A,
$$

denote its traceless part. Moreover, observe that the second equation in (1.10) is invariant under changes $H \mapsto H h$, where $h$ is a central gauge transformation. Therefore, by standard Hodge theory, it suffices to solve only for the traceless part of the first equation. With this understood, we state

Proposition 3.3. (Sufficient Conditions) Suppose that $\left(E_{1}, E\right)$ is a locallyfree, saturated, $\tau$-stable extension pair with respect to the holomorphic structure $D^{\prime \prime}$ on $E$. Let $K$ be a hermitian metric on $E$ of determinant $\chi$. Then 
496 Georgios Daskalopoulos, Karen Uhlenbeck and Richard Wentworth

there is a unique $H \in \operatorname{Met}_{2}^{p}(\chi)$ such that

$$
\left\{\begin{array}{l}
\widehat{\Lambda F}_{D^{\prime \prime}, H}-\sqrt{-1} \tau \pi_{H}+\sqrt{-1} \frac{R_{1}}{R} \tau \mathbf{I}=0 \\
\pi_{H}^{\perp} D^{\prime \prime} \pi_{H}=0,
\end{array}\right.
$$

where $\pi_{H}$ is the projection operator onto $E_{1}$ corresponding to $H$.

The proof of Proposition 3.3 will occupy the next two subsections.

\subsection{The modified Donaldson Lagrangian.}

Let $E_{1}, E$ be as before, and let $\mathbb{G}\left(R_{1}, E\right)$ be the associated Grassmann bundle on $X$ as in $\S 1.3$. Over $\mathbb{G}\left(R_{1}, E\right)$ there is the relative anti-tautological line bundle $\gamma \rightarrow \mathbb{G}\left(R_{1}, E\right)$. A hermitian metric on $E$ endows $\gamma$ with a hermitian metric as well.

Definition 3.4. Given $(E, K)$, a hermitian bundle as above, let

$$
S=S(K)=\left\{s \in \text { End } E: s=s^{*_{K}}\right\},
$$

and

$$
\hat{S}=\hat{S}(K)=\{s \in S: \operatorname{tr} s(x)=0 \text { for all } x \in X\} .
$$

Definition 3.5. Let $H \in \operatorname{Met}_{2}^{p}(\chi)$. Let $s \in L_{2}^{p}(\hat{S})$ be the unique gauge transformation such that $H=K e^{s}$. Let $f: X \rightarrow \mathbb{G}\left(R_{1}, E\right)$ be the section corresponding to the weak subbundle $E_{1}$, and let

$$
\nu_{x}\left(f(x), e^{s(x) / 2}\right)=\log \frac{\left\|e^{s(x) / 2} \widehat{f(x)}\right\|_{\gamma_{x}}^{2}}{\|\widehat{f(x)}\|_{\gamma_{x}}^{2}}
$$

be the relative version of $\nu$ defined in $\S 1.2$. Set

$$
N(K, H)=\int_{X} \nu_{x}\left(f(x), e^{s(x) / 2}\right) d \operatorname{Vol}(x) .
$$

Lemma 3.6. $N(K, H)$ converges.

Proof. By Lemma 1.12, $\nu_{x}\left(f(x), e^{s(x) / 2}\right) \geq 2 \pi \operatorname{tr}\left(s(x) \cdot \pi^{f(x)}\right)$. Since both $s(x), \pi^{f(x)} \in L^{\infty}, \nu_{x}\left(f(x), e^{s(x) / 2}\right)$ is bounded from below by and $L^{\infty}$ function. On the other hand, if $\rho, \imath$ are as in $\S 1.2$,

$$
\log \frac{\left\|e^{s(x) / 2} \widehat{f(x)}\right\|_{\gamma_{x}}^{2}}{\|\widehat{f(x)}\|_{\gamma_{x}}^{2}}=\log \frac{\| \rho\left(e^{\left.s(x) / 2) \widehat{\imath f(x)} \|_{\mathbb{C}^{(R}}^{2}\right)}\right.}{\|\widehat{\imath f(x)}\|_{\mathbb{C}^{(R}\left(R_{1}\right)}^{2}} \leq C(s(x)),
$$


where $C$ depends continuously on $s(x)$. Since $s \in L_{2}^{p} \subset L^{\infty}, \nu_{x}\left(f(x), e^{s(x) / 2}\right)$ is bounded above by an $L^{\infty}$ function as well, and the lemma follows.

Let $M_{D}(K, H)$ be the usual Donaldson functional (cf. [Do] or [S1]). Set

$$
M_{\tau}(K, H)=M_{D}(K, H)+\frac{\tau}{\pi} N(K, H) .
$$

Then $M_{\mathcal{T}}(K, H)$ is our modified Donaldson Lagrangian. The following lists the important properties of $M_{\tau}$ :

Proposition 3.7. Set

$$
m_{\tau}(H)=\widehat{\Lambda F}_{D^{\prime \prime}, H}-\sqrt{-1} \tau \pi_{H}+\sqrt{-1} \frac{R_{1}}{R} \tau \mathbf{I} .
$$

Then the following hold:

(i) $\frac{d}{d t} M_{\tau}\left(K, K e^{t s}\right)=2 \sqrt{-1} \int_{X} \operatorname{tr}\left(s m_{\tau}\left(K e^{t s}\right)\right)$;

(ii) For $\tau \geq 0, \frac{d^{2}}{d t^{2}} M_{\tau}\left(K, K e^{t s}\right) \geq 0$;

(iii) If $\left(E_{1}, E\right)$ is simple, then $\frac{d^{2}}{d t^{2}} M_{\tau}\left(K, K e^{t s}\right)=0$ if and only is $s=0$;

(iv) $M_{\tau}(K, H)+M_{\tau}(H, J)=M_{\tau}(K, J)$.

Proof. (i) We have

$$
\begin{aligned}
\frac{d}{d t} M_{\tau}\left(K, K e^{t s}\right) & =\frac{d}{d t}\left\{M_{D}\left(K, K e^{t s}\right)+\frac{\tau}{\pi} \int_{X} \nu_{x}\left(f(x), e^{t s(x) / 2}\right) d \operatorname{Vol}(x)\right\} \\
& =2 \sqrt{-1} \int_{X} \operatorname{tr} s \Lambda F_{K e^{t s}}+2 \tau \int_{X} \operatorname{tr} s \pi_{K e^{s}} \\
& =2 \sqrt{-1} \int_{X} \operatorname{tr} s m_{\tau}\left(K e^{s}\right) .
\end{aligned}
$$

The second equality follows from [S1, proof of 5.2.1], (1.12), and Lemma 1.14. The third equality follows because $s$ is traceless. (ii) Immediate from [S1, proof of 5.2.1] and (1.12). (iii) Assume the equality. By (i), (ii), Proposition 1.18, and the fact that $s$ is traceless, we obtain $\frac{d}{d t} \mu_{\tau}\left(D^{\prime \prime}, \pi\right) \cdot s=$ 0 . Note that $\mu_{\tau}$ given in (1.19) is still the moment map for the action of $L_{2}^{p}(\mathfrak{g})$ on $L_{1}^{p}(\mathcal{A}) \times L_{1}^{p}\left(\mathbb{G}\left(R_{1}, E\right)\right)$. Then by general arguments concerning moment maps (cf. $[\mathrm{Ki}, 3.7]),\left(D^{\prime \prime}, \pi_{K}\right)$ is fixed by the one-parameter subgroup $e^{t s}$. Since $\left(D^{\prime \prime}, \pi^{K}\right)$ is simple, this implies $s=0$. (iv) Let $H=K e^{s_{1}}, J=H e^{s_{2}}$, where $s_{1} \in L_{2}^{p}(\hat{S}(K)), s_{2} \in L_{2}^{P}(\hat{S}(H))$. It suffices to show that pointwise:

$$
\frac{\left\|e^{s_{1}(x) / 2} \hat{V}\right\|_{\gamma(K)}^{2}}{\|\hat{V}\|_{\gamma(K)}^{2}} \cdot \frac{\left\|e^{s_{2}(x) / 2} \hat{V}\right\|_{\gamma(H)}^{2}}{\|\hat{V}\|_{\gamma(H)}^{2}}=\frac{\left\|e^{s_{1}(x)+s_{2}(x) / 2} \hat{V}\right\|_{\gamma(K)}^{2}}{\|\hat{V}\|_{\gamma(K)}^{2}} .
$$


498 Georgios Daskalopoulos, Karen Uhlenbeck and Richard Wentworth

But

$$
\left\|e^{s_{2}(x) / 2} \hat{V}\right\|_{\gamma(H)}^{2}=\left\langle e^{s_{2}(x) / 2} \hat{V}, e^{s_{1}(x)} e^{s_{2}(x) / 2} \hat{V}\right\rangle_{\gamma(K)}=\left\|e^{s_{1}(x)+s_{2}(x) / 2} \hat{V}\right\|_{\gamma(K)}^{2},
$$

and similarly, $\|\hat{V}\|_{\gamma(H)}^{2}=\left\|e^{t s_{1}(x) / 2} \hat{V}\right\|_{\gamma(K)}^{2}$. By taking the logarithm and integrating over $X$, we have $N(K, H)+N(H, J)=N(K, J)$. The rest follows as in [Do, Proposition 6].

\subsection{Proof of the theorem.}

Given $B>0$, we set

$$
M e t_{2, B}^{p}(\chi)=\left\{H \in M e t_{2}^{p}(\chi):\left\|m_{\tau}(H)\right\|_{L^{p}, H}^{p} \leq B\right\} .
$$

Proposition 3.8. If $\left(E_{1}, E\right)$ is simple (see Definition 2.10) and $H$ minimizes $M(K, \cdot)$ in $\operatorname{Met}_{2, B}^{p}(\chi)$, then $m_{\tau}(H)=0$.

Proof. (cf. [S1, 5.3.1] and [Br, 3.4.2]) By [S1, 5.1.2.1], if $s \in L_{2}^{p}\left(S^{\perp}\right)$ and $H_{t}=H e^{t s}$, then

$$
\left.\frac{d}{d t}\right|_{t=0} m_{\tau}\left(H_{t}\right)=L(s)
$$

where

$$
L(s)=\Lambda D^{\prime \prime} D_{H}^{\prime}(s)-\left.\sqrt{-1} \tau \frac{d}{d t}\right|_{t=0} \pi_{H_{t}} .
$$

Now assume $m_{\tau}(H) \neq 0$ and that we can find $s \neq 0$ so that $L(s)=-m_{\tau}(H)$. Then as in $[\mathrm{Br}, 3.4 .2]$ we obtain

$$
\left.\frac{d}{d t}\right|_{t=0}\left\|m_{\tau}\left(H_{t}\right)\right\|_{L^{p}, H_{t}}^{p}=-\frac{p}{2}\left\|m_{\tau}(H)\right\|_{L^{p}, H}^{p} \leq 0 .
$$

Moreover, since $\left(E_{1}, E\right)$ is simple, by Proposition 3.7 we obtain

$$
\begin{aligned}
0 & <\left.\frac{d^{2}}{d t^{2}}\right|_{t=0} M_{\tau}\left(K, H_{t}\right)=\left.\frac{d}{d t}\right|_{t=0} \int_{X} \operatorname{tr} s m_{\tau}\left(H_{t}\right) \\
& =\int_{X} \operatorname{tr} s \cdot L(s)=-\int_{X} \operatorname{tr} s m_{\tau}(H)=-\left.\frac{d}{d t}\right|_{t=0} M_{\tau}\left(K, H_{t}\right),
\end{aligned}
$$

hence,

$$
\left.\frac{d}{d t}\right|_{t=0} M_{\tau}\left(K, H_{t}\right)<0
$$


This contradicts the assumption that $H$ minimizes $M_{\tau}(\cdot, K)$ and the proposition will follow provided we can solve $L(s)=-m_{\tau}(H)$. But

$$
\sqrt{-1} L(s)=\Delta_{H}^{\prime}(s)+\left.\tau \frac{d}{d t}\right|_{t=0} \pi_{H_{t}}
$$

which is an elliptic, self-adjoint operator. Ellipticity is clear, and selfadjointness follows since $\left.\frac{d}{d t}\right|_{t=0} \pi_{H_{t}}$ is self-adjoint. The latter holds because for $H_{t}=H e^{t s},\left(\pi_{H_{t}}\right)^{*_{H}}=e^{-\bar{t} s}\left(\left(\pi_{H_{t}}\right)^{*_{H}} e^{t s}=\pi_{H_{t}}\right.$, hence

$$
\left.\frac{d}{d t}\right|_{t=0}\left(\pi_{H_{t}}\right)^{*_{H_{t}}}=\left.\frac{d}{d t}\right|_{t=0}\left(\pi_{H_{t}}\right)^{*_{H}} .
$$

Now

$$
\left(\left.\frac{d}{d t}\right|_{t=0} \pi_{H_{t}}\right)^{*_{H}}=\left.\frac{d}{d t}\right|_{t=0}\left(\pi_{H_{t}}\right)^{*_{H}}=\left.\frac{d}{d t}\right|_{t=0}\left(\pi_{H_{t}}\right)^{*_{H_{t}}}=\left.\frac{d}{d t}\right|_{t=0} \pi_{H_{t}} .
$$

Now since $\sqrt{-1} L(s)$ is self-adjoint and elliptic, it suffices to show that $L$ has no kernel. But

$$
\begin{aligned}
L(s)=0 \Rightarrow \int_{X} \operatorname{tr} s L(s)=0 \Rightarrow \\
\left.\quad \frac{d}{d t}\right|_{t=0} \int_{X} \operatorname{tr} s m_{\tau}\left(H_{t}\right)=\left.0 \Rightarrow \frac{d^{2}}{d t^{2}}\right|_{t=0} M_{\tau}\left(K, H_{t}\right)=0 .
\end{aligned}
$$

Hence, again by the simplicity of $\left(E_{1}, E\right), s=0$.

Proposition 3.9 (Main estimate). There are constants $C_{1}, C_{2}>0$ such that for all $H=K e^{s} \in M e t_{2, B}^{p}(\chi)$,

$$
\sup |s| \leq C_{1} M_{\tau}\left(K, K e^{s}\right)+C_{2},
$$

or else $\left(E_{1}, E\right)$ is not stable.

Proof. Since the proof closely parallels [S1], we shall only give a sketch: First, as in [S1, 6.2] we have an equivalence between $C^{0}$. and $L^{1}$ bounds. Suppose that the main estimate does not hold. Then as in [S1, 6.3] we can choose a sequence of constants $C_{i} \rightarrow \infty, s_{i} \in \hat{S}$, such that $\left\|s_{i}\right\|_{L^{1}} \rightarrow \infty$ and $\left\|s_{i}\right\|_{L^{1}} \geq C_{i} M_{\tau}\left(K e^{s_{i}}\right)$. Set $\ell_{i}=\left\|s_{i}\right\|_{L^{1}}, u_{i}=\ell_{i}^{-1} s_{i}$, so that $\left\|u_{i}\right\|_{L^{1}}=1$, and $\sup \left|u_{i}\right| \leq C$. We have the following: 
500 Georgios Daskalopoulos, Karen Uhlenbeck and Richard Wentworth

Lemma 3.10. After passing to a subsequence, $u_{i} \rightarrow u_{\infty}$ weakly in $L_{1}^{2}(\hat{S})$. Moreover, if $F: \mathbb{R} \times \mathbb{R} \rightarrow \mathbb{R}$ is a positive, smooth function such that $F(x, y) \leq$ $1 /(x-y)$ whenever $x>y$, then with the notation as in [S1, 6.3.3],

$$
\int_{X}\left\langle F\left(u_{\infty}\right) D^{\prime \prime} u_{\infty}, D^{\prime \prime} u_{\infty}\right\rangle_{K}+\sqrt{-1} \int_{X} \operatorname{tr} u_{\infty} \Lambda F_{K}+\tau \int_{X} \operatorname{tr} u_{\infty} \cdot \pi_{K} \leq 0 .
$$

Proof. We first claim that

$$
\int_{X}\left\langle F\left(u_{i}\right) D^{\prime \prime} u_{i}, D^{\prime \prime} u_{i}\right\rangle_{K}+\sqrt{-1} \int_{X} \operatorname{tr} u_{i} \Lambda F_{K}+\tau \int_{X} \operatorname{tr} u_{i} \cdot \pi_{K} \leq \frac{1}{2} C_{i}^{-1} .
$$

Indeed, by combining the inequalities in $[\mathrm{S} 1,6.3 .3]$ with Lemma 1.13,

$$
\begin{array}{rl}
\frac{\ell_{i}}{C_{i}} \geq M_{\tau}\left(K, K e^{\ell_{i} u_{i}}\right) \geq 2 & 2 \ell_{i}\left\{\int_{X}\left\langle F\left(u_{i}\right) D^{\prime \prime} u_{i}, D^{\prime \prime} u_{i}\right\rangle_{K}+\sqrt{-1} \int_{X} \operatorname{tr} u_{i} \Lambda F_{K}\right\} \\
& +2 \ell_{i} \tau \int_{X} \operatorname{tr} u_{i} \pi_{K},
\end{array}
$$

which proves the claim. The rest is as in [S1, 6.3.3].

Now we conclude the proof of Proposition 3.9 by showing that $\left(E_{1}, E\right)$ is not stable. First, as in [S1, Lemma 6.3.4], the eigenvalues of $u_{\infty}$ are constant. Let $\lambda_{1}, \ldots, \lambda_{r}$ denote the distinct eigenvalues of $u_{\infty}$, listed in ascending order, and as in $[\mathrm{S} 1,6.4 .2]$ let $p_{j}: \mathbb{R} \rightarrow \mathbb{R}, j=1, \ldots, r-1$ be smooth, positive functions with $p_{j}(x)=1$ if $x \leq \lambda_{j}$ and $p_{j}(x)=0$ if $x \geq \lambda_{j+1}$. Set $\pi_{j}=p_{j}\left(u_{\infty}\right)$ be the $L_{1}^{2}$-subbundle of $E$. By the UhlenbeckYau regularity result (cf. [UY]), $\pi_{j}$ represents a saturated subsheaf of $E$. Set $\alpha_{j}=\lambda_{j}-\lambda_{j-1}$, and write

$$
u_{\infty}=\lambda_{r} \mathbf{I}-\sum_{j=1}^{r} \alpha_{j} \pi_{j} .
$$

Since $\operatorname{tr} u_{\infty}=0, \lambda_{r} R-\sum \alpha_{j} \operatorname{rk}\left(\pi_{j}\right)=0$. If $\left(E_{1}, E\right)$ were $\tau$-stable, then we would have

$$
\mu\left(\pi_{j}\right)+\tau \frac{\operatorname{rk}\left(E_{1} \cap \pi_{j}\right)}{\operatorname{rk} \pi_{j}}<\mu+\tau \frac{R_{1}}{R},
$$

and hence,

$$
\begin{aligned}
& \lambda_{r} R\left(\mu+\tau \frac{R_{1}}{R}\right)-\sum \alpha_{j} \operatorname{rk}\left(\pi_{j}\right)\left(\mu\left(\pi_{j}\right)+\tau \frac{\operatorname{rk}\left(\pi_{j} \cap E_{1}\right)}{\operatorname{rk} \pi_{j}}\right)>0 \\
& \Rightarrow \lambda_{r} c_{1}(E)+\lambda_{r} \tau R_{1}-\sum \alpha_{j} c_{1}\left(\pi_{j}\right)-\tau \sum \alpha_{j} \operatorname{rk}\left(\pi_{j} \cap E_{1}\right)>0
\end{aligned}
$$


Therefore,

$$
Q=\lambda_{r} c_{1}(E)-\sum \alpha_{j} c_{1}\left(\pi_{j}\right)+\tau\left(\lambda_{r} R_{1}-\sum \alpha_{j} \operatorname{rk}\left(\pi_{j} \cap E_{1}\right)\right)>0 .
$$

But then,

$$
\begin{aligned}
0<2 \pi Q=\sqrt{-1} & \int_{X} \operatorname{tr} u_{\infty} \Lambda F_{K} \\
& +\int_{X}\left\langle\sum\left(\alpha_{j} \tilde{P}_{j}^{2}\right)\left(u_{\infty}\right) D^{\prime \prime} u_{\infty}, D^{\prime \prime} u_{\infty}\right\rangle_{K}+\tau \int_{X} \operatorname{tr} u_{\infty} \pi_{K} .
\end{aligned}
$$

This contradicts Lemma 3.10, and concludes the proof of Proposition 3.3 (the uniqueness of $H$ follows as in [S1] by the convexity of $M_{\tau}$ ). This also concludes the proof of Theorem 3.1.

\subsection{Bogomolov inequality.}

As an application of Theorem 3.1 we have the following version of the Bogomolov inequality for stable vector bundles:

Theorem 3.11. Suppose that $\left(E_{1}, E\right)$ is a locally-free, saturated, $\tau$-stable extension pair on Kähler manifold with Kähler form $\omega$. Then

$$
c_{2}(E) \wedge \omega^{n-2} \geq \frac{R-1}{2 R} c_{1}(E)^{2} \wedge \omega^{n-2}-\tau^{2} \frac{R_{1}\left(R-R_{1}\right)}{2 R} \omega^{n} .
$$

Proof. By Theorem 3.1 there is a hermitian metric $H$ on $E$ such that the compatible connection gives a solution to equations (1.10). Since the curvature is in $L^{2}$ and the connection is gauge equivalent to a smooth connection, we may use the Chern-Weil theory to compute $c_{1}$ and $c_{2}$. Almost everywhere, we can choose a local orthonormal trivialization $\left\{e_{1}, \ldots, e_{R_{1}}, e_{R_{1}+1}, \ldots, e_{R}\right\}$ for $E$ compatible with $\pi_{H}$. Then from (1.10),

$$
\begin{aligned}
\left|\sqrt{-1} \Lambda F_{D^{\prime \prime}, H}\right|^{2} & =\sum_{i, j}\left|\left(\sqrt{-1} \Lambda F_{D^{\prime \prime}, H}\right)_{i j}\right|^{2} \\
& =R_{1}\left(\left(\frac{R_{1}}{R} \tau+\mu\right)-\tau\right)^{2}+\left(R-R_{1}\right)\left(\frac{R_{1}}{R} \tau+\mu\right)^{2} \\
& =R \mu^{2}+\tau^{2} \frac{R_{1}}{R}\left(R-R_{1}\right)
\end{aligned}
$$

or,

$$
R\left|\sqrt{-1} \Lambda F_{D^{\prime \prime}, H}\right|^{2}=\operatorname{tr}\left(\sqrt{-1} \Lambda F_{D^{\prime \prime}, H}\right)^{2}+\tau^{2} \frac{R_{1}}{R}\left(R-R_{1}\right)
$$


502 Georgios Daskalopoulos, Karen Uhlenbeck and Richard Wentworth

The result then follows as in $[K o$, p. 114].

\section{Moduli Space Construction.}

The purpose of this section is prove the following

Theorem 4.1. The moduli space of $\tau$-stable smooth extension pairs $\left(E_{1}, E\right)$ satisfying the condition $H^{2}\left(X, E n d_{0} E\right)=0$, where $\operatorname{End}_{0} E$ denotes the sheaf of traceless endomorphisms of $E$, has the structure of a Kähler manifold.

In $\S 4.1$ we will discuss the deformation theory. In $\S 4.2$ we will put the complex structure on the moduli space, and in $\$ 4.3$ we will define a compatible symplectic structure. For the rest of this section, let $E$ denote a smooth vector bundle on $X$ and $E_{1}$ a subbundle of $E$. By $\tau$-stable, in this section, we shall mean $\tau$-slope stable. Also, while we will deal with spaces of smooth sections of bundles, to justify the use of the inverse and implicit function theorems used repeatedly in $\S 4.2$ and $\S 4.3$, one must consider appropriate Sobolev completions. Since this is fairly standard (see [Ko], for example) we will suppress this point and leave the reader to fill in the analytic details.

\subsection{The deformation complex.}

Fix a smooth pair $\left(E_{1}, E\right)$. Let $D^{\prime \prime}$ denote the holomorphic structure on $E$ and $\pi$ the projection operator onto $E_{1}$ with respect to the background hermitian metric $K$. In other words, $\pi=\pi^{*}=\pi^{2}, \pi^{\perp} D^{\prime \prime} \pi=0, \pi(E)=E_{1}$. Set $\pi^{\perp}(E)=E_{2}$. Consider the following complex:

$\left(C_{D^{\prime \prime}, \pi}\right)$

$$
\begin{aligned}
& 0 \longrightarrow \Omega^{0}(\text { End } E) \stackrel{d_{0}}{\longrightarrow} \Omega^{0,1}(\operatorname{End} E) \oplus \Omega^{0}\left(\operatorname{Hom}\left(E_{1}, E_{2}\right)\right) \stackrel{d_{1}}{\longrightarrow} \\
& \longrightarrow \Omega^{0,2}(\text { End } E) \oplus \Omega^{0,1}\left(\operatorname{Hom}\left(E_{1}, E_{2}\right)\right) \stackrel{d_{2}}{\longrightarrow} \\
& \longrightarrow \Omega^{0,3}(\text { End } E) \oplus \Omega^{0,2}\left(\operatorname{Hom}\left(E_{1}, E_{2}\right)\right) \stackrel{d_{3}}{\longrightarrow} \\
& \cdots \stackrel{d_{n-1}}{\longrightarrow} \Omega^{0, n}(\text { End } E) \oplus \Omega^{0, n-1}\left(\operatorname{Hom}\left(E_{1}, E_{2}\right)\right) \stackrel{d_{n}}{\longrightarrow} \\
& \longrightarrow \Omega^{0, n}\left(\operatorname{Hom}\left(E_{1}, E_{2}\right)\right) \longrightarrow 0 .
\end{aligned}
$$

The differentials above are defined as follows:

$$
\begin{array}{ll}
d_{0}(u) & =\left(-D^{\prime \prime} u, \pi^{\perp} u \pi\right) \\
d_{i}(\alpha, \eta) & =\left(-D^{\prime \prime} \alpha,\left(D_{1}^{\prime \prime}\right)^{*} \otimes D_{2}^{\prime \prime} \eta+\pi^{\perp} \alpha \pi\right), \quad i>0,
\end{array}
$$

where $D_{1}^{\prime \prime}, D_{2}^{\prime \prime}$ are the induced holomorphic structures on $E_{1}, E_{2}$. Equivalently, if we denote also by $\eta$ the extension of $\eta$ by 0 as an endomorphism of 
$E$, then

$$
d_{i}(\alpha, \eta)=\left(-D^{\prime \prime} \alpha, \pi^{\perp}\left(D^{\prime \prime} \eta+\alpha\right) \pi\right), \quad i>0 .
$$

Finally, a third description of $d_{1}$ will be useful later:

$$
d_{1}(\alpha, \eta)=\left(-D^{\prime \prime} \alpha,-\eta D^{\prime \prime} \pi+\pi^{\perp} D^{\prime \prime} \eta+\pi^{\perp} \alpha \pi\right) .
$$

The verification of the equivalence of (4.3) and (4.4) is a direct calculation.

We now prove the following

Proposition 4.5. (i) $C_{D^{\prime \prime}, \pi}$ is an elliptic complex.

(ii) if $\left(D^{\prime \prime}, \pi\right)$ is simple, then $H^{0}\left(C_{D^{\prime \prime}, \pi}\right) \simeq \mathbb{C}$.

Proof. (i) We first check that it is a complex.

$$
\begin{aligned}
d_{1} d_{0} & =d_{1}\left(-D^{\prime \prime} u, \pi^{\perp} u \pi\right)=\left(\left(D^{\prime \prime}\right)^{2} u, \pi^{\perp}\left(D^{\prime \prime}\left(\pi^{\perp} u \pi\right)-D^{\prime \prime} u\right) \pi\right) \\
& =\left(\left(D^{\prime \prime}\right)^{2} u,-\pi^{\perp}\left(D^{\prime \prime} \pi\right) u \pi+\pi^{\perp}\left(D^{\prime \prime} u\right) \pi+\pi^{\perp} u\left(D^{\prime \prime} \pi\right) \pi-\pi^{\perp}\left(D^{\prime \prime} u\right) \pi\right) \\
& =(0,0),
\end{aligned}
$$

since $\left(D^{\prime \prime}\right)^{2}=0, \pi^{\perp} D^{\prime \prime} \pi=0$, and $\left(D^{\prime \prime} \pi\right) \pi=0$.

$$
\begin{aligned}
d_{i+1} d_{i}(\alpha, \eta) & =d_{i+1}\left(-D^{\prime \prime} \alpha, \pi^{\perp}\left(D^{\prime \prime} \eta+\alpha\right) \pi\right) \\
& =\left(\left(D^{\prime \prime}\right)^{2} \alpha, \pi^{\perp}\left(D^{\prime \prime}\left(\pi^{\perp}\left(D^{\prime \prime} \eta+\alpha\right) \pi\right) \pi-\pi^{\perp} D^{\prime \prime} \alpha \pi\right)\right. \\
& =\left(\left(D^{\prime \prime}\right)^{2} \alpha,-\pi^{\perp} D^{\prime \prime} \pi\left(D^{\prime \prime} \eta+\alpha\right) \pi+\pi^{\perp}\left(\left(D^{\prime \prime}\right)^{2} \eta+D^{\prime \prime} \alpha\right) \pi\right. \\
& \left.\quad+(-1)^{i} \pi^{\perp}\left(D^{\prime \prime} \eta+\alpha\right) D^{\prime \prime} \pi \pi-\pi^{\perp} D^{\prime \prime} \alpha \pi\right) \\
& =(0,0),
\end{aligned}
$$

for the same reasons as above. Thus, $C_{D^{\prime \prime}, \pi}$ is a complex. Ellipticity follows directly from the ellipticity of the Dolbeault complex. (ii) is clear from Definition 2.10.

\subsection{Complex analytic structure.}

We use the deformation complex described in the previous subsection to put a complex structure on the moduli space of simple, smooth pairs. Let $\mathcal{A}^{1,1}$ be the space of integrable complex structures in $\mathcal{A}$. Under the assumption $H^{2}\left(X, \operatorname{End}_{0} E^{D^{\prime \prime}}\right)=0$, an argument as in [Ko, Theorem 3.2.3] 
504 Georgios Daskalopoulos, Karen Uhlenbeck and Richard Wentworth

shows that $\mathcal{A}^{1,1}$ is smooth at $D^{\prime \prime}$. Indeed, the Kuranishi map (cf. [Ko, VII, 3.10]) defines a biholomorphism of a neighborhood of $D^{\prime \prime}$ in $\mathcal{A}^{1,1}$ and a neighborhood of 0 in the vector space

$$
\left\{\alpha \in \Omega^{0,1}(\text { End } E): D^{\prime \prime} \alpha=0\right\} \text {. }
$$

Let $\mathcal{H} \subset \mathcal{A}^{1,1} \times \Omega^{0}\left(\mathbb{G}\left(R_{1}, E\right)\right)$ be the subspace defined in (1.19). Thus, $\mathcal{H}$ consists of pairs $\left(D^{\prime \prime}, \pi\right)$ such that $\left(D^{\prime \prime}\right)^{2}=0$ and $\pi^{\perp} D^{\prime \prime} \pi=0$.

Notation 4.6. Let $\mathcal{H}^{*}$ be the space of simple extension pairs, and $\mathcal{H}^{0}$ the subspace of $\mathcal{H}^{*}$ consisting of pairs $\left(D^{\prime \prime}, \pi\right)$ such that $H^{2}\left(X, \operatorname{End}_{0} E^{D^{\prime \prime}}\right)=0$.

Lemma 4.7. (i) $\mathcal{H}^{*}$ is open in $\mathcal{H}$ and $\mathcal{H}^{0}$ is open in $\mathcal{H}^{*}$. (ii) $\mathcal{H}^{0}$ is a smooth manifold.

Proof. (i) Follows by semicontinuity of cohomology (cf. [Kod, Corollary of Theorem 7.8]). (ii) Let $\mathcal{E} \rightarrow \Omega^{0}\left(\mathbb{G}\left(R_{1}, E\right)\right)$ be the vector bundle defined in [DU, §2] whose fiber over $\pi \in \Omega^{0}\left(\mathbb{G}\left(R_{1}, E\right)\right)$ is $\Omega^{0}\left(\operatorname{Hom}\left(\pi(E), \pi^{\perp}(E)\right)\right)$. Let $\mathcal{A}_{0}^{1,1}$ be the subspace of $\mathcal{A}^{1,1}$ consisting of those $D^{\prime \prime}$ such that $H^{2}\left(X, \operatorname{End}_{0} E^{D^{\prime \prime}}\right)=0$, and let

$$
\mathcal{D}: \mathcal{A}_{0}^{1,1} \times \Omega^{0}\left(\mathbb{G}\left(R_{1}, E\right)\right) \longrightarrow \mathcal{E}
$$

be the map defined by $\mathcal{D}\left(D^{\prime \prime}, \pi\right)=\pi^{\perp} D^{\prime \prime} \pi$. Then $\mathcal{D}$ is a fiber bundle over $\Omega^{0}\left(\mathbb{G}\left(R_{1}, E\right)\right)$, and by (4.3) and (4.4), $\mathcal{D}$ is transversal to the zero section $0_{\mathcal{E}}$ of $\mathcal{E}$. It follows (but see the warning at the beginning of this section) that $\mathcal{D}^{-1}\left(0_{\mathcal{E}}\right)$ is a smooth manifold, and thus $\mathcal{D}^{-1}\left(0_{\mathcal{E}}\right) \cap \mathcal{H}^{*}=\mathcal{H}^{0}$ is also a smooth manifold.

Now fix $\left(D^{\prime \prime}, \pi\right) \in \mathcal{H}^{*}$. Set $E_{1}=\pi(E), E_{2}=\pi^{\perp}(E)$. Let

$$
\exp _{\pi}: \Omega^{0}\left(\operatorname{Hom}\left(E_{1}, E_{2}\right)\right) \longrightarrow \mathbb{G}\left(R_{1}, E\right)
$$

be the exponential map defined in a neighborhood of $0 \in \Omega^{0}\left(\operatorname{Hom}\left(E_{1}, E_{2}\right)\right)$ generalizing the pointwise exponential map for $\mathbb{G}\left(R_{1}, R\right)$. Let

$$
\begin{gathered}
f: \Omega^{0,1}(\text { End } E) \times \Omega^{0}\left(\operatorname{Hom}\left(E_{1}, E_{2}\right)\right) \longrightarrow \Omega^{0,2}(\operatorname{End} E) \times \Omega^{0,1}\left(\operatorname{Hom}\left(E_{1}, E_{2}\right)\right) \\
f(\alpha, \eta)=\left(D^{\prime \prime} \alpha+\alpha \wedge \alpha, \mathcal{D}\left(D^{\prime \prime}+\alpha, \exp _{\pi} \eta\right)\right),
\end{gathered}
$$

and define

$$
\begin{aligned}
\mathcal{S}_{D^{\prime \prime}, \pi}=\left\{(\alpha, \eta) \in \Omega^{0,1}(\text { End } E) \times \Omega^{0}\left(\operatorname{Hom}\left(E_{1}, E_{2}\right)\right):\right. & \\
& \left.f(\alpha, \eta)=0, d_{0}^{*}(\alpha, \eta)=0\right\} .
\end{aligned}
$$


Lemma 4.9. If $\left(D^{\prime \prime}, \pi\right) \in \mathcal{H}^{0}$, then $\mathcal{S}_{D^{\prime \prime}, \pi}$ is a smooth manifold in a neighborhood of $(0,0)$, and $T_{(0,0)} \mathcal{S}_{D^{\prime \prime}, \pi} \simeq H^{1}\left(C_{D^{\prime \prime}, \pi}\right)$.

Proof. Define $\hat{\mathcal{S}}_{D^{\prime \prime}, \pi}=\{(\alpha, \eta): f(\alpha, \eta)=0\}$. T'he map

$$
h: \hat{\mathcal{S}}_{D^{\prime \prime}, \pi} \longrightarrow \mathcal{H}^{0}:(\alpha, \eta) \mapsto\left(D^{\prime \prime}+\alpha, \exp _{\pi} \eta\right)
$$

defines a biholomorphism of a neighborhood of $(0,0) \in \hat{\mathcal{S}}_{D^{\prime \prime}, \pi}$ onto a neighborhood of $\left(D^{\prime \prime}, \pi\right) \in \mathcal{H}^{0}$, and therefore $\hat{\mathcal{S}}_{D^{\prime \prime}, \pi}$ is smooth in a neighborhood of $\left(D^{\prime \prime}, \pi\right)$ by Lemma 4.7. Let $\mathcal{P}$ be the complement in $\Omega^{0}$ (End $\left.E\right)$ of $H^{0}\left(C_{D^{\prime \prime}, \pi}\right) \simeq \mathbb{C}$ with respect to the $L^{2}$-inner product, and consider the map

$$
H: \hat{\mathcal{S}}_{D^{\prime \prime}, \pi} \longrightarrow \mathcal{P}:(\alpha, \eta) \mapsto d_{0}^{*}(\alpha, \eta) .
$$

Then under the identification $T_{(0,0)} \hat{\mathcal{S}}_{D^{\prime \prime}, \pi} \simeq \operatorname{ker} d_{1}$, the map $d H_{(0,0)}$ is $d_{0}^{*}$. Since $H^{0}\left(C_{D^{\prime \prime}, \pi}\right)=\mathbb{C}$, it follows that $d_{0}^{*}$ is onto, and the inverse function theorem for the map $H$ completes the proof.

Now fix $\left(D^{\prime \prime}, \pi\right)$ as before and define the Kuranishi map

$$
\begin{gathered}
k: \Omega^{0,1}(\text { End } E) \times \Omega^{0}\left(\operatorname{Hom}\left(E_{1}, E_{2}\right)\right) \longrightarrow \Omega^{0,1}(\operatorname{End} E) \oplus \Omega^{0}\left(\operatorname{Hom}\left(E_{1}, E_{2}\right)\right) \\
(\alpha, \eta) \mapsto(\alpha, \eta)+d_{1}^{*} G\left(f(\alpha, \eta)-d_{1}(\alpha, \eta)\right),
\end{gathered}
$$

where $G$ is the Green's function for $C_{D^{\prime \prime}, \pi}$. By the Hodge decomposition for $C_{D^{\prime \prime}, \pi}$ it follows that

$$
\begin{array}{ll}
d_{1} k(\alpha, \eta) & =0 \\
d_{1}^{*} k(\alpha, \eta) & =d_{1}^{*}(\alpha, \eta)
\end{array}
$$

We claim

Lemma 4.11. Assume $\left(D^{\prime \prime}, \pi\right) \in \mathcal{H}^{*}$. Then the map

$$
\rho: \mathcal{S}_{D^{\prime \prime}, \pi} \longrightarrow \mathcal{H}^{*} / \mathfrak{G}^{\mathbb{C}}:(\alpha, \eta) \mapsto\left[D^{\prime \prime}+\alpha, \exp _{\pi} \eta\right]
$$

is a local homeomorphism of a neighborhood of $(0,0) \in \mathcal{S}_{D^{\prime \prime}, \pi}$ onto a neighborhood of $\left[D^{\prime \prime}, \pi\right] \in \mathcal{H}^{*} / \mathfrak{G}^{\mathbb{C}}$.

Proof. (cf. [Ko, VII, Theorem 3.17]) We prove surjectivity first. It suffices to show that given

$$
\left(D^{\prime \prime}+\beta, \exp _{\pi} \nu\right) \in \mathcal{H}^{*}, \quad(\beta, \nu) \in \Omega^{0,1}(\operatorname{End} E) \times \Omega^{0}\left(\operatorname{Hom}\left(E_{1}, E_{2}\right)\right)
$$


sufficiently close to $(0,0)$, there is a $g \in \mathfrak{G}^{\mathbb{C}}$ such that $\left(D^{\prime \prime}+\alpha, \exp _{\pi} \eta\right)=$ $g\left(D^{\prime \prime}+\beta, \exp _{\pi} \nu\right)$ satisfies $d_{1}^{*}(\alpha, \eta)=0$ and $f(\alpha, \eta)=0$. The second equation is automatically satisfied because $\mathcal{H}^{*}$ is gauge invariant. For the first equation, let $\mathcal{P}$ be as in the proof of Lemma 4.9 and consider the map

$$
\begin{gathered}
P: \mathcal{P} \times \Omega^{0,1}(\text { End } E) \times \Omega^{0}\left(\operatorname{Hom}\left(E_{1}, E_{2}\right)\right) \longrightarrow \mathcal{P} \\
(u,(\beta, \nu)) \mapsto d_{0}^{*}\left(e^{u}\left(D^{\prime \prime}+\beta\right)-D^{\prime \prime}, \exp _{\pi}^{-1}\left(e^{u}\left(\exp _{\pi}(\nu)\right)\right)\right) .
\end{gathered}
$$

The map $P$ is well-defined in a neighborhood of $(0,0,0)$, and

$$
\frac{\partial P}{\partial u}(0,0,0)=d_{0}^{*} d_{0}: \mathcal{P} \longrightarrow \mathcal{P}
$$

is invertible. Hence, by the implicit function theorem (again, note the warning) there is a function $(w,(\beta, \nu)) \mapsto u=u(w,(\beta, \nu))$ defined in a neighborhood of $(0,0) \in \Omega^{0,1}(\operatorname{End} E) \times \Omega^{0}\left(\operatorname{Hom}\left(E_{1}, E_{2}\right)\right)$ such that $P(u(w,(\beta, \nu)),(\beta, \nu))=w$. By setting $g=e^{u},(\alpha, \eta)=$ $\left(g\left(D^{\prime \prime}\right)-D^{\prime \prime}, \exp _{\pi}^{-1}\left(g \exp _{\pi} \nu\right)\right)$ satisfies $d_{0}^{*}(\alpha, \eta)=0$. To show that $\rho$ is injective around $(0,0)$ it suffices to prove that if $g\left(D^{\prime \prime}+\alpha_{1}, \exp _{\pi} \eta_{1}\right)=$ $\left(D^{\prime \prime}+\alpha_{2}, \exp _{\pi} \eta_{2}\right)$ with $\left(\alpha_{1}, \eta_{1}\right),\left(\alpha_{2}, \eta_{2}\right) \in \mathcal{S}_{D^{\prime \prime}, \pi}$ sufficiently close to $(0,0)$, then $\left(\alpha_{1}, \eta_{1}\right)=\left(\alpha_{2}, \eta_{2}\right)$. But we can ignore the second factors and apply [Ko, Theorem VII, 3.17] to $D^{\prime \prime}+\alpha_{1}$ and $D^{\prime \prime}+\alpha_{2}$ to show $g$ is close to the identity. The rest then follows by the implicit function theorem for the map $P$.

We are ready to prove

Proposition 4.12. The moduli space $\mathcal{H}^{*} / \mathfrak{G}^{\mathbb{C}}$ of simple pairs is a (possibly non-Hausdorff and non-reduced) complex analytic space. Moreover, $\mathcal{H}^{0} / \mathfrak{G}^{\mathbb{C}}$ is a smooth complex manifold with

$$
T_{D^{\prime \prime}, \pi} \mathcal{H}^{0} / \mathfrak{G}^{\mathbb{C}} \simeq H^{1}\left(C_{D^{\prime \prime}, \pi}\right) .
$$

Proof. (cf. [Ko, VII, 3.23, 3.31, and 3.32]) Since the derivative of the Kuranishi map $k$ at $(0,0)$ is the identity map, $k$ defines according to $(4.10)$ an injective, holomorphic map

$$
k: \mathcal{S}_{D^{\prime \prime}, \pi} \longrightarrow H^{1}\left(C_{D^{\prime \prime}, \pi}\right)
$$

defined in a neighborhood of $(0,0)$. The first statement in the proposition follows as in [Ko, VII, 3.32]. The second part follows by Lemmas 4.9 and 4.11 , and the fact that (4.13) is an injective local immersion. 


\subsection{Kähler structure.}

We now turn to the issue of describing a Kähler structure on the moduli space of extension pairs and providing a proof of Theorem 4.1.

Notation 4.14. Let $\mathcal{H}_{\tau} \subset \mathcal{H}^{*}$ denote the subspace of $\tau$-stable extension pairs $\left(D^{\prime \prime}, \pi\right)$ in $\mathcal{H}^{*}$. Let $\mathcal{H}_{\tau}^{0}=\mathcal{H}_{\tau} \cap \mathcal{H}^{0}$.

Lemma 4.15. $\mathcal{H}_{\tau}$ is open in $\mathcal{H}^{*}$ and $\mathcal{H}_{\tau}^{0}$ is open in $\mathcal{H}_{\tau}$. Moreover, $\mathcal{H}_{\tau}^{0}$ is a smooth manifold.

Proof. In view of Lemma 4.7, it suffices to show that $\mathcal{H}_{\tau}$ is open in $\mathcal{H}^{*}$. By Theorem 3.1 we must show that if $\left(D^{\prime \prime}, \pi\right)$ satisfies equations (1.10) and $\left(\widetilde{D}^{\prime \prime}, \tilde{\pi}\right)$ is sufficiently close to $\left(D^{\prime \prime}, \pi\right)$, then there is a $g \in \mathfrak{G}^{\mathbb{C}}$ such that $g\left(\widetilde{D}^{\prime \prime}, \tilde{\pi}\right)$ satisfies $(1.10)$ as well. This is a standard application of the implicit function theorem (cf. [Ko, VII, Theorem 4.21]). Let $\hat{S}$ be as in Definition 3.4 , and define the map

$$
\begin{gathered}
Q: \Omega^{0}(\hat{S}) \times \mathcal{H}^{*} \longrightarrow \hat{S} \\
Q\left(u, \widetilde{D}^{\prime \prime}, \tilde{\pi}\right)=\widehat{\Lambda F}_{\widetilde{D}^{\prime \prime}, K}-\sqrt{-1} \tau \tilde{\pi}+\sqrt{-1} \frac{R_{1}}{R} \tau \mathbf{I} .
\end{gathered}
$$

By the calculation in Proposition 3.8,

$$
\left.\frac{\partial Q}{\partial u}\right|_{\left(\mathbf{I}, D^{\prime \prime}, \pi\right)}(s)=L(2 s)
$$

where $L$ is an elliptic, self-adjoint operator with trivial kernel. By the implicit function theorem, we can solve the equation $Q\left(u\left(\widetilde{D}^{\prime \prime}, \tilde{\pi}\right), \widetilde{D}^{\prime \prime}, \tilde{\pi}\right)=0$ for $\left(\widetilde{D}^{\prime \prime}, \tilde{\pi}\right)$ near $\left(D^{\prime \prime}, \pi\right)$. Hence, $e^{u}\left(\widetilde{D}^{\prime \prime}, \tilde{\pi}\right)$ satisfies $(1.10)$, and by Theorem 3.1 and standard Hodge theory, $\left(\widetilde{D}^{\prime \prime}, \tilde{\pi}\right)$ is also $\tau$-stable. This proves the openness of $\mathcal{H}_{\tau}$.

We now consider $\mathcal{H}_{\tau}^{0}$ as a $\overline{\mathfrak{G}}=\mathfrak{G} / U(1)$-space. Let

$$
\begin{gathered}
\bar{\mu}_{\tau}: \mathcal{H}_{\tau}^{0} \longrightarrow(\operatorname{Lie} \overline{\mathfrak{G}})^{*} \\
\bar{\mu}_{\tau}=\mu_{\tau}+\sqrt{-1}\left(\frac{R_{1}}{R} \tau+\mu\right) \mathbf{I},
\end{gathered}
$$

where $\mu_{\tau}$ is the moment map defined in (1.18). 
508 Georgios Daskalopoulos, Karen Uhlenbeck and Richard Wentworth

Lemma 4.16. (i) $\bar{\mu}_{\tau}$ is an Ad-equivariant moment map for the $\overline{\mathfrak{G}}$-action. (ii) Zero is a regular value of $\bar{\mu}_{\tau}$. (iii) Given $\left(D^{\prime \prime}, \pi\right) \in \bar{\mu}_{\tau}^{-1}(0)$, there is a submanifold $\mathcal{F}_{D^{\prime \prime}, \pi}$ of $\mu_{\tau}^{-1}(0)$ such that

$$
T_{D^{\prime \prime}, \pi} \mu_{\tau}^{-1}(0) \simeq T_{D^{\prime \prime}, \pi}\left(\mathcal{F}_{D^{\prime \prime}, \pi}\right) \oplus T_{D^{\prime \prime}, \pi}\left(\overline{\mathfrak{G}}\left(D^{\prime \prime}, \pi\right)\right) .
$$

Proof. (i) Since $\int_{X} \bar{\mu}_{\tau}\left(D^{\prime \prime}, \pi\right)=0$, the result follows from (1.18). (ii) This follows from general considerations about moment maps (cf. [GS, §2]) and the fact that $\overline{\mathfrak{G}}$ acts freely on $\mathcal{H}_{\tau}^{0}$. (iii) Fix $\left(D^{\prime \prime}, \pi\right) \in \bar{\mu}_{\tau}^{-1}(0)$ and consider the complex $\left(\widetilde{C}_{D^{\prime \prime}, \pi}\right)$

$$
\begin{aligned}
& 0 \longrightarrow \Omega^{0}(\operatorname{ad} E) \stackrel{\tilde{d}_{0}}{\longrightarrow} \Omega^{0,1}(\text { End } E) \oplus \Omega^{0}\left(\operatorname{Hom}\left(E_{1}, E_{2}\right)\right) \stackrel{\tilde{d}_{1}}{\longrightarrow} \\
& \longrightarrow \Omega^{0}(\operatorname{ad} E) \oplus \Omega^{0,2}(\text { End } E) \oplus \Omega^{0,1}\left(\operatorname{Hom}\left(E_{1}, E_{2}\right)\right) \stackrel{\tilde{d}_{2}}{\longrightarrow} \\
& \longrightarrow \Omega^{0,3}(\text { End } E) \oplus \Omega^{0,2}\left(\operatorname{Hom}\left(E_{1}, E_{2}\right)\right) \stackrel{\tilde{d}_{3}}{\longrightarrow} \\
& \quad \stackrel{\tilde{d}_{n-1}}{\longrightarrow} \Omega^{0, n}(\text { End } E) \oplus \Omega^{0, n-1}\left(\operatorname{Hom}\left(E_{1}, E_{2}\right)\right) \stackrel{\tilde{d}_{n}}{\longrightarrow} \\
& \longrightarrow \Omega^{0, n}\left(\operatorname{Hom}\left(E_{1}, E_{2}\right)\right) \longrightarrow 0 .
\end{aligned}
$$

In the above, $E_{1}=\pi(E), E_{2}=\pi^{\perp}(E)$, and

$$
\begin{array}{ll}
\tilde{d}_{0}(u)=\left(D^{\prime \prime} u, \pi^{\perp} u \pi\right), & \tilde{d}_{1}(\alpha, \eta)=\left(\left(d \mu_{\tau}\right)_{D^{\prime \prime}, \pi}(\alpha, \eta), d_{1}(\alpha, \eta)\right) \\
\tilde{d}_{2}(\xi, \alpha, \eta)=d_{2}(\alpha, \eta), & \tilde{d}_{i}(\alpha, \eta)=d_{i}(\alpha, \eta), \quad i>2 .
\end{array}
$$

It is easily checked (cf. proof of Proposition 4.5) that $\widetilde{C}_{D^{\prime \prime}, \pi}$ is an elliptic complex. Let

$$
\begin{aligned}
\widetilde{\mathcal{F}}_{D^{\prime \prime}, \pi}=\left\{(\alpha, \eta) \in \Omega^{0,1}(\text { End } E) \times \Omega^{0}\left(\operatorname{Hom}\left(E_{1}, E_{2}\right)\right): f(\alpha, \eta)=0,\right. \\
\left.d \bar{\mu}_{\tau}(\alpha, \eta)=0, \quad \tilde{d}_{0}^{*}(\alpha, \eta)=0\right\} .
\end{aligned}
$$

It follows as in Lemma 4.9 that $\widetilde{\mathcal{F}}_{D^{\prime \prime}, \pi}$ is a smooth manifold in a neighborhood of $(0,0)$, and

$$
T_{D^{\prime \prime}, \pi} \widetilde{\mathcal{F}}_{D^{\prime \prime}, \pi} \simeq H^{1}\left(\widetilde{C}_{D^{\prime \prime}, \pi}\right) .
$$

Let $\mathcal{F}_{D^{\prime \prime}, \pi} \subset \mu_{\tau}^{-1}(0)$ be defined by

$$
\mathcal{F}_{D^{\prime \prime}, \pi}=\left\{\left(D^{\prime \prime}+\alpha, \exp _{\pi} \eta\right):(\alpha, \eta) \in \widetilde{\mathcal{F}}_{D^{\prime \prime}, \pi}\right\} .
$$

Clearly, $\mathcal{F}_{D^{\prime \prime}, \pi}$ is a smooth submanifold of $\bar{\mu}_{\tau}^{-1}(0)$ around $\left(D^{\prime \prime}, \pi\right)$, and

$$
T_{D^{\prime \prime}, \pi} \mathcal{F}_{D^{\prime \prime}, \pi} \simeq H^{1}\left(\widetilde{C}_{D^{\prime \prime}, \pi}\right)
$$


The Lemma follows since $T_{D^{\prime \prime}, \pi} \mu_{\tau}^{-1}(0)=\operatorname{ker} \tilde{d}_{1}$, and $T_{D^{\prime \prime}, \pi}\left(\overline{\mathfrak{G}}\left(D^{\prime \prime}, \pi\right)\right)=$ $\operatorname{Im} \tilde{d}_{0}$.

We are now ready to prove Theorem 4.1.

Proposition 4.17. The symplectic form $\omega_{\tau}$ define in (1.17) descends to a symplectic form $\hat{\omega}_{\tau}$ on $\mu_{\tau}^{-1}(0) / \mathfrak{G} \simeq \mathcal{H}_{\tau}^{0} / \mathfrak{G}^{\mathbb{C}}$. With respect to the complex structure, $\hat{\omega}_{\tau}$ is Kähler.

Proof. The first part of the proposition follows by Lemma 4.16 and the infinite dimensional version of Marsden-Weinstein reduction (cf. [Ko, VII, 5.8]). In order to show that $\hat{\omega}_{\tau}$ is a Kähler form, fix $X, Y \in T_{D^{\prime \prime}, \pi}^{1,0}\left(\mathcal{H}_{\tau}^{0} / \mathfrak{G}^{\mathbb{C}}\right)$. Under the identification

$$
T_{D^{\prime \prime}, \pi}^{1,0}\left(\mathcal{H}_{\tau}^{0} / \mathfrak{G}^{\mathbb{C}}\right) \simeq H^{1}\left(C_{D^{\prime \prime}, \pi}\right) \simeq H^{1}\left(\widetilde{C}_{D^{\prime \prime}, \pi}\right) \simeq T_{D^{\prime \prime}, \pi}\left(\mathcal{F}_{D^{\prime \prime}, \pi}\right)
$$

write $X=(\alpha, \eta), Y=(\beta, \xi)$. Then $\hat{\omega}_{\tau}(X, Y)=\omega_{\tau}((\alpha, \eta),(\beta, \xi))=0$, since $(\alpha, \eta),(\beta, \xi) \in T^{1,0} \mathcal{H}_{\tau}^{0}$, and $\omega_{\tau}$ is a Kähler form.

\section{Geometric Invariant Theory.}

We shall now present a construction of a moduli space parametrizing equivalence classes of $\tau$-Gieseker semistable torsion-free quotient pairs on an algebraic manifold $X$. Since the method used will be geometric invariant theory applied to the action of a reductive algebraic group on a projective scheme, the moduli space will inherit a projective scheme structure. In particular, this gives a compactification of the moduli of smooth extension pairs constructed in the previous section.

The arguments that follow very closely parallel those of Simpson, and we refer the reader to [S2] for more details. Throughout this section, $X$ will denote a smooth projective variety of complex dimension $n$.

\subsection{Embedding into the Quot scheme.}

Notation 5.1. Let $h_{E}$ and $h_{F}$ be polynomials of degree $n$. We denote by $\mu_{\tau}\left(h_{E}, h_{F}\right)$ the set of all $\tau$-slope-semistable quotients $E \rightarrow F \rightarrow 0$, where $E$ is a torsion-free sheaf with Hilbert polynomial $h_{E}$ and $F$ is a coherent sheaf with Hilbert polynomial $h_{F}$. Similarly, we denote by $S_{\tau}\left(h_{E}, h_{F}\right)$ the set of $\tau$-Gieseker semistable quotients. 
510 Georgios Daskalopoulos, Karen Uhlenbeck and Richard Wentworth

The first step in the GIT construction is a boundedness result:

Proposition 5.2. The set $\mu_{\tau}\left(h_{E}, h_{F}\right)$ (and hence also $S_{\tau}\left(h_{E}, h_{F}\right)$ ) is bounded, i.e. parametrized by a scheme of finite type over $\mathbb{C}$.

Proof. Slope-semistability implies that for every subsheaf $E^{\prime} \subset E$ we have

$$
\mu\left(E^{\prime}\right) \leq \mu(E)+\tau\left(\frac{\mathrm{rk} F^{\prime}}{\mathrm{rk} E^{\prime}}-\frac{\mathrm{rk} F}{\mathrm{rk} E}\right) \leq \mu(E)+\tau,
$$

so by [Ma] we have that the set of such $E$ is bounded. Then for fixed $E$, the set of all quotients $F$ with Hilbert polynomial $h_{F}$ is bounded by [Gr]. The result follows.

We now wish to embed all the quotients $q \in S_{\tau}\left(h_{E}, h_{F}\right)$ into a product of Quot schemes. Recall that we have fixed an ample line bundle $A$ on $X$. By the boundedness result above, there exists an integer $K_{0}$ such that for all $k \geq K_{0}$ and $q \in S_{\tau}\left(h_{E}, h_{F}\right)$, we can write

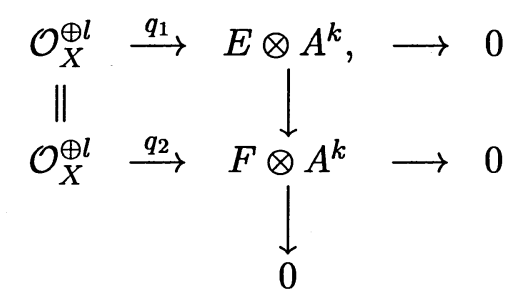

Let $\operatorname{Quot}\left(\mathcal{O}_{X}^{\oplus l}, h_{E, k}\right)$ and $\operatorname{Quot}\left(\mathcal{O}_{X}^{\oplus l}, h_{F, k}\right)$ denote the Grothendieck Quot schemes parametrizing quotients of $\mathcal{O}_{X}^{\oplus l}$ with Hilbert polynomials $h_{E, k}(j)=$ $h_{E}(k+j)$ and $h_{F, k}(j)=h_{F}(k+j)$, respectively. Furthermore, we let $\widehat{Q}_{k}$ denote the closed subscheme of

$$
\operatorname{Quot}\left(\mathcal{O}_{X}^{\oplus l}, h_{E, k}\right) \times \operatorname{Quot}\left(\mathcal{O}_{X}^{\oplus l}, h_{F, k}\right)
$$

defined by the condition $\operatorname{ker} q_{1} \subset \operatorname{ker} q_{2}$. Let $Q_{k} \subset \widehat{Q}_{k}$ be the subscheme where $E$ is torsion-free, and denote its closure by $\bar{Q}_{k} \subset \widehat{Q}_{k}$. Let $\pi_{E}, \pi_{F}$ denote the projections onto the first and second factors of (5.4). We have universal quotients:

$$
\begin{array}{ll}
\mathcal{O}_{X \times Q u o t}^{\oplus l}\left(\mathcal{O}_{X}^{\oplus l}, h_{E, k}\right) & \longrightarrow U_{E} \longrightarrow 0 \\
\mathcal{O}_{X \times Q u o t}^{\oplus l}\left(\mathcal{O}_{X}^{\oplus l, h_{F}, k}\right) & \longrightarrow U_{F} \longrightarrow 0
\end{array}
$$


on $X \times \operatorname{Quot}\left(\mathcal{O}_{X}^{\oplus l}, h_{E, k}\right)$ and $X \times \operatorname{Quot}\left(\mathcal{O}_{X}^{\oplus l}, h_{F, k}\right)$. It follows that for the quotients

$$
\mathcal{O}_{X \times \widehat{Q}_{k}}^{\oplus l} \stackrel{\hat{q}_{1}}{\longrightarrow} \pi_{E}^{*} U_{E} \longrightarrow 0, \quad \mathcal{O}_{X \times \widehat{Q}_{k}}^{\oplus l} \stackrel{\hat{q}_{2}}{\longrightarrow} \pi_{F}^{*} U_{F} \longrightarrow 0,
$$

we have $\operatorname{ker} \hat{q}_{1} \subset \operatorname{ker} \hat{q}_{2}$; hence, on $X \times \widehat{Q}_{k}$ we have a universal quotient

$$
\pi_{E}^{*} U_{E} \longrightarrow \pi_{F}^{*} U_{F} \longrightarrow 0 \text {. }
$$

We have shown that to every point $E \rightarrow F \rightarrow 0$ in $S_{\tau}\left(h_{E}, h_{F}\right)$ there corresponds a closed point $\hat{q} \in Q_{k} \subset \bar{Q}_{k} \subset \widehat{Q}_{k}$ such that the universal quotient (5.5) restricted to $X \times\{\hat{q}\}$ is isomorphic to $E \otimes A^{k} \longrightarrow F \otimes A^{k} \longrightarrow 0$.

By tensoring with a further high power of $A$ we can embed $\bar{Q}_{k}$ into a product of Grassmannians

$$
G\left(V \otimes W, h_{E}(k+j)\right) \times G\left(V \otimes W, h_{F}(k+j)\right)
$$

of $h_{E}(k+j)$ and $h_{F}(k+j)$ dimensional quotients of $V \simeq \mathbb{C}^{l} \simeq H^{0}\left(\mathcal{O}_{X}^{\oplus l}\right)$ tensor $W \simeq H^{0}\left(A^{j}\right)$. Now there is the diagonal action of $S L(V)$ on (5.6), and the image of $\bar{Q}_{k}$ is invariant under this action. We linearize the action by the $S L(V)$ equivariant Plücker embedding into a product of projective spaces. Let $\mathcal{O}(r, s)$ denote the line bundle on (5.6) obtained by pulling back appropriate powers of hyperplanes. Then $S L(V)$ acts on $\mathcal{O}(r, s)$. Also, let

$$
k_{A}=\frac{1}{n} \underbrace{A \cdots A}_{n} .
$$

Since GIT semistability is invariant upon taking powers, it makes sense to consider linearizations of the action of $S L(V)$ on rational line bundles. So for rational values of $\tau$, we choose the linearization

$$
\mathcal{O}\left(k k_{A}+M, \tau\right) \text {. }
$$

Here, $M$ is an integer which we shall fix presently.

Our goal then is to show that the GIT-semistable points in $\bar{Q}_{k}$ with respect to the linearization (5.8) correspond to $\tau$-Gieseker semistable quotients. The main result is Theorem 5.18 below. Before carrying this out, we consider two preliminary details:

First, the choice of $M$. Among the quotients $E \rightarrow F \rightarrow 0$ in $S_{\tau}\left(h_{E}, h_{F}\right)$ consider those for which there exists a subsheaf $E^{\prime} \subset E$ with $\mu_{\tau}^{Q}\left(E^{\prime}, F^{\prime}\right)=$ $\mu_{\tau}^{Q}(E, F)$. We may assume that $E^{\prime}$ has torsion-free quotient. Then the set of such $E^{\prime}$ is bounded. In particular, the set of Hilbert polynomials of such $E^{\prime}$ 
512 Georgios Daskalopoulos, Karen Uhlenbeck and Richard Wentworth

is finite. Let $C$ be the maximum over the absolute values of the coefficients of $k^{n-2} /(n-2)$ ! in the polynomials $p\left(E^{\prime}, k\right)-p(E, k)$ as $E^{\prime}$ runs over the family described above. We then choose the integer $M$ such that

$$
\left(M+\tau \frac{\operatorname{rk} F}{\operatorname{rk} E}-\mu(E)\right) \frac{\tau}{(\operatorname{rk} E)^{2}}-k_{A}(n-1) C \geq 1 .
$$

Notice that $M$ depends only on $h_{E}, h_{F}, \tau$, and of course $A$.

Second, we need a criterion for semistability in Grassmannians:

Lemma 5.10. Consider the action of $S L(V)$ on the product

$$
G\left(V \otimes W, R_{1}\right) \times G\left(V \otimes W, R_{2}\right)
$$

of $R_{1}$ and $R_{2}$ dimensional quotients of $V \otimes W$, and linearize the action on $\mathcal{O}(r, s)$ with respect to the Plücker embedding. Then a point $q=\left(q_{1}, q_{2}\right)$ is semistable (resp. stable) if and only if for all non-zero linear subspaces $H \subset V$ we have

$$
\begin{aligned}
\left(r R_{1}+s R_{2}\right) \operatorname{dim} H \leq(r \operatorname{dim} & \pi_{1}(H \otimes W) \\
& \left.+s \operatorname{dim} \pi_{2}(H \otimes W)\right) \operatorname{dim} V \quad(\text { resp. } \quad<)
\end{aligned}
$$

where $\pi_{1}$ and $\pi_{2}$ denote the projections onto the first and second factors of (5.11).

Proof. See [GIT] or $[\mathrm{KgNw}]$.

\subsection{Comparison of stability conditions.}

We now prove a sequence of lemmas necessary to establish the correspondence between $\tau$-Gieseker semistable quotients and GIT semistable points.

Lemma 5.12. For fixed $k$, there exists a $J$ such that for all $j \geq J$ the following holds: If $\hat{q}=\left(q_{1}, q_{2}, q\right) \in \bar{Q}_{k}$ (see (5.3)) is such that the induced map

$$
V \simeq \mathbb{C}^{l} \simeq H^{0}\left(\mathcal{O}_{X}^{\oplus l}\right) \longrightarrow H^{0}\left(E \otimes A^{k}\right)
$$


is an isomorphism, and for all $H \subset \mathbb{C}^{l}$ where the induced subsheaf $E_{H}$ is non-zero we have

$$
\begin{gathered}
{\left[\left(k k_{A}+M\right) \chi\left(E \otimes A^{k+j}\right)+\tau \chi\left(F \otimes A^{k+j}\right] h^{0}\left(E_{H} \otimes A^{k}\right)\right.} \\
\leq\left[\left(k k_{A}+M\right) \chi\left(E_{H} \otimes A^{k+j}\right)+\tau \chi\left(F_{H} \otimes A^{k+j}\right] h^{0}\left(E \otimes A^{k}\right) \quad(\text { resp. } \quad<)\right.
\end{gathered}
$$

then the point $\hat{q}$ is semistable with respect to the linearization $\mathcal{O}\left(k k_{A}+M, \tau\right)$ on $\bar{Q}_{k}$.

Proof. Notice first that $\operatorname{dim} H \leq h^{0}\left(E_{H} \otimes A^{k}\right)$. Let $H$ vary in $V$ and $\hat{q}$ vary in $\bar{Q}_{k}$. Then by boundedness, the families of kernels

$$
\begin{aligned}
& 0 \longrightarrow K_{E_{H}} \longrightarrow H \otimes \mathcal{O}_{X} \stackrel{q_{1}}{\longrightarrow} E_{H} \otimes A^{k} \longrightarrow 0 \\
& 0 \longrightarrow K_{F_{H}} \longrightarrow H \otimes \mathcal{O}_{X} \stackrel{q_{2}}{\longrightarrow} F_{H} \otimes A^{k} \longrightarrow 0
\end{aligned}
$$

are bounded. Therefore, we may choose $J$ such that for $j \geq J$,

$$
\begin{array}{ll}
h^{1}\left(K_{E_{H}} \otimes A^{j}\right) & =h^{1}\left(K_{F_{H}} \otimes A^{j}\right)=0 \\
h^{0}\left(E_{H} \otimes A^{k}\right) & \leq h^{0}\left(E_{H} \otimes A^{k+j}\right)=\chi\left(E_{H} \otimes A^{k+j}\right) \\
h^{0}\left(F_{H} \otimes A^{k}\right) & \leq h^{0}\left(F_{H} \otimes A^{k+j}\right)=\chi\left(F_{H} \otimes A^{k+j}\right)
\end{array}
$$

We get

$$
\left\{\begin{array}{l}
H \otimes H^{0}\left(A^{j}\right) \longrightarrow H^{0}\left(E_{H} \otimes A^{k+j}\right) \longrightarrow 0 \\
H \otimes H^{0}\left(A^{j}\right) \longrightarrow H^{0}\left(F_{H} \otimes A^{k+j}\right) \longrightarrow 0
\end{array}\right.
$$

so

$$
\begin{array}{ll}
\operatorname{dim} \pi_{1}(H \otimes W) & =h^{0}\left(E_{H} \otimes A^{k+j}\right) \\
\operatorname{dim} \pi_{2}(H \otimes W) & =h^{0}\left(F_{H} \otimes A^{k+j}\right) .
\end{array}
$$

Since $E_{H} \neq 0$, the first of these is non-zero for large $j$. Hence,

$$
\begin{aligned}
{\left[\left(k k_{A}+M\right)\right.} & \left.h_{E}(k+j)+\tau h_{F}(k+j)\right] \operatorname{dim} H \\
& =\left[\left(k k_{A}+M\right) \chi\left(E \otimes A^{k+j}\right)+\tau \chi\left(F \otimes A^{k+j}\right] \operatorname{dim} H\right. \\
& \leq\left[\left(k k_{A}+M\right) \chi\left(E \otimes A^{k+j}\right)+\tau \chi\left(F \otimes A^{k+j}\right] h^{0}\left(E_{H} \otimes A^{k}\right)\right. \\
& \leq\left[\left(k k_{A}+M\right) \chi\left(E_{H} \otimes A^{k+j}\right)+\tau \chi\left(F_{H} \otimes A^{k+j}\right] h^{0}\left(E \otimes A^{k}\right)\right. \\
& =\left[\left(k k_{A}+M\right) \operatorname{dim} \pi_{1}(H \otimes W)+\tau \operatorname{dim} \pi_{2}(H \otimes W)\right] \operatorname{dim} V,
\end{aligned}
$$

so the result follows from Lemma 5.10. 
514 Georgios Daskalopoulos, Karen Uhlenbeck and Richard Wentworth

Lemma 5.13. There is a $J$ such that for all $j \geq J$ the following holds: If $\hat{q} \in \bar{Q}_{k}$ is GIT semistable with respect to the linearization $\mathcal{O}\left(k k_{A}+M, \tau\right)$, then the homomorphism

$$
V \simeq H^{0}\left(\mathcal{O}_{X}^{\oplus l}\right) \longrightarrow H^{0}\left(E \otimes A^{k}\right)
$$

is injective, and for any non-zero linear subspace $H \subset V$, we have

$$
\left(\left(k k_{A}+M\right) \mathrm{rk} E+\tau \mathrm{rk} F\right) \operatorname{dim} H \leq\left(\left(k k_{A}+M\right) \mathrm{rk} E_{H}+\tau \mathrm{rk} F_{H}\right) \operatorname{dim} V .
$$

Moreover, if $E \otimes A^{k}$ is the sheaf associated to $\hat{q}$, then for every quotient

$$
E \otimes A^{k} \longrightarrow E^{\prime \prime} \otimes A^{k} \longrightarrow 0
$$

we have

$$
\begin{aligned}
\left(\left(k k_{A}+M\right) \operatorname{rk} E+\tau \operatorname{rk} F\right) h^{0}\left(E^{\prime \prime} \otimes A^{k}\right) & \\
& \geq\left(\left(k k_{A}+M\right) \operatorname{rk} E^{\prime \prime}+\tau \operatorname{rk} F^{\prime \prime}\right) \operatorname{dim} V,
\end{aligned}
$$

where $F^{\prime \prime}$ is defined so as to make the following diagram commute:

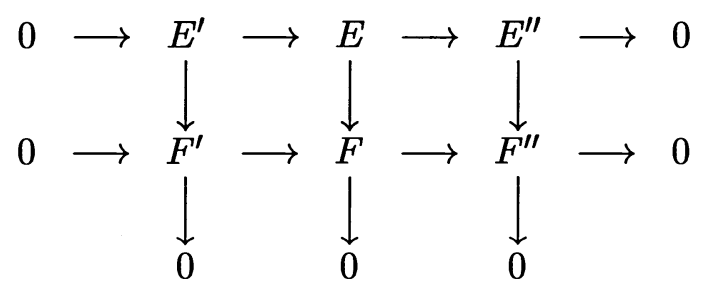

Proof. Let $\hat{q} \in \bar{Q}_{k}$ be semistable. Then Lemma 5.10 implies that for non-zero $H \subset V$,

$$
\operatorname{dim} \pi_{1}(H \otimes W)+\operatorname{dim} \pi_{2}(H \otimes W) \neq 0,
$$

where $\pi_{1}$ and $\pi_{2}$ are the projections

$$
\begin{array}{ll}
V \otimes H^{0}\left(A^{j}\right) & \stackrel{\pi_{1}}{\longrightarrow} H^{0}\left(E \otimes A^{k+j}\right) \longrightarrow 0 \\
V \otimes H^{0}\left(A^{j}\right) & \stackrel{\pi_{2}}{\longrightarrow} H^{0}\left(F \otimes A^{k+j}\right) \longrightarrow 0 .
\end{array}
$$

Both maps factor through $H^{0}\left(E \otimes A^{k}\right) \otimes H^{0}\left(A^{j}\right)$ and $H^{0}\left(F \otimes A^{k}\right) \otimes H^{0}\left(A^{j}\right)$, respectively. Moreover, the map $\pi_{2}$ factors through $H^{0}\left(E \otimes A^{k+j}\right)$, because the point is in $\widehat{Q}_{k}$. In particular, $\operatorname{dim} \pi_{1}(H \otimes W) \neq 0$. Since $H$ is arbitrary, we obtain the desired injectivity. Now suppose to the contrary that

$$
\left(\left(k k_{A}+M\right) \mathrm{rk} E+\tau \mathrm{rk} F\right) \operatorname{dim} H>\left(\left(k k_{A}+M\right) \operatorname{rk} E_{H}+\tau \operatorname{rk} F_{H}\right) \operatorname{dim} V .
$$


Then for $n$ large,

$$
\begin{gathered}
\left(\left(k k_{A}+M\right) \chi\left(E \otimes A^{k+j}\right)+\tau \chi\left(F \otimes A^{k+j}\right)\right) \operatorname{dim} H \\
\leq\left(\left(k k_{A}+M\right) \chi\left(E_{H} \otimes A^{k+j}\right)+\tau \chi\left(F_{H} \otimes A^{k+j}\right)\right) \operatorname{dim} V .
\end{gathered}
$$

Since $H$ runs over a bounded family, we can choose $J$ such that for all $j \geq J$ the above inequality holds, and moreover such that

$$
\begin{aligned}
& h^{0}\left(E_{H} \otimes A^{k+j}\right)=\chi\left(E_{H} \otimes A^{k+j}\right)=\operatorname{dim} \pi_{1}(H \otimes W) \\
& h^{0}\left(F_{H} \otimes A^{k+j}\right)=\chi\left(F_{H} \otimes A^{k+j}\right)=\operatorname{dim} \pi_{2}(H \otimes W) \text {. }
\end{aligned}
$$

But this contradicts Lemma 5.10.

Lemma 5.14. There is a $K$ such that for all $k \geq K$ the following is true: If $E \rightarrow F \rightarrow 0$ is a $\tau$-Gieseker semistable quotient, then for all subquotients $E^{\prime} \rightarrow F^{\prime} \rightarrow 0$ we have:

$\left(\left(k k_{A}+M\right) \operatorname{rk} E+\tau \operatorname{rk} F\right) h^{0}\left(E^{\prime} \otimes A^{k}\right) \leq\left(\left(k k_{A}+M\right) \operatorname{rk} E^{\prime}+\tau \operatorname{rk} F^{\prime}\right) h_{E}(k)$

Moreover, if equality holds, we have

(i) $h^{0}\left(E^{\prime} \otimes A^{k}\right)=\chi\left(E^{\prime} \otimes A^{k}\right)$;

(ii) $\mathrm{rk} F^{\prime} / \mathrm{rk} E^{\prime}=\mathrm{rk} F / \mathrm{rk} E$;

(iii) For all $j, p\left(E^{\prime}, j\right)=p(E, j)$;

(iv) For $j>>0, p\left(F^{\prime}, j\right) \geq p(F, j)$.

Proof. Since the set of $E$ is bounded, fix one. If $E^{\prime}$ is a subsheaf of $E$, let $R^{\prime}=\operatorname{rk} E^{\prime}$, and let $Q_{i}\left(R_{i}=\operatorname{rk} Q_{i}, \mu_{i}=\mu\left(Q_{i}\right)\right)$ denote the terms in its $A$-Harder-Narasimhan filtration. We have

$$
\begin{aligned}
h^{0}\left(E^{\prime} \otimes A^{k}\right) & \leq \sum_{i} h^{0}\left(Q_{i} \otimes A^{k}\right) \\
\mu_{i} & \leq \mu+\tau \\
\sum_{i} R_{i} & =R^{\prime}
\end{aligned}
$$

For $k$ large we have (see [S2]):

$$
h^{0}\left(Q_{i} \otimes A^{k}\right) \leq \frac{k_{A} R_{i}}{n !}\left(\left(\mu_{i} / k_{A}\right)+k+B\right)^{n}
$$


516 Georgios Daskalopoulos, Karen Uhlenbeck and Richard Wentworth

for some $B$. Let $\nu=\min \mu_{i}$. Then

$$
\begin{aligned}
& h^{0}\left(E^{\prime} \otimes A^{k}\right) \\
\leq & \left.\frac{k_{A}\left(R^{\prime}-1\right)}{n !}\left(\left(\mu(E) / k_{A}\right)+k+B\right)+\tau / k_{A}\right)^{n}+\frac{k_{A}}{n !}\left(\left(\nu / k_{A}\right)+k+B\right)^{n} .
\end{aligned}
$$

For any $\beta$ there exists a $C \geq \beta$ such that if $\nu \leq \mu(E)-C$ the sum above is $\leq\left(k_{A} R / n !\right)(k-\beta)^{n}$. We can also choose $\beta$ such that for large $k$,

$$
\frac{k_{A}}{n !}(k-\beta)^{n}<p(E, k)\left(1-\frac{\tau}{k k_{A}+M}\right) .
$$

Then for the appropriate $C$ and $\nu \leq \mu(E)-C$, we have

$$
\begin{aligned}
h^{0}\left(E^{\prime} \otimes A^{k}\right) & <\left(\mathrm{rk} E^{\prime}\right) p(E, k)\left(1-\frac{\tau}{k k_{A}+M}\right) \\
& \leq\left(\mathrm{rk} E^{\prime}\right) p(E, k)\left(1+\frac{\tau}{k k_{A}+M} \frac{\mathrm{rk} F^{\prime}}{\mathrm{rk} E^{\prime}}\right)\left(1+\frac{\tau}{k k_{A}+M} \frac{\mathrm{rk} F}{\mathrm{rk} E}\right)^{-1}
\end{aligned}
$$

which implies

$\left(\left(k k_{A}+M\right) \operatorname{rk} E+\tau \operatorname{rk} F\right) h^{0}\left(E^{\prime} \otimes A^{k}\right)<\left(\left(k k_{A}+M\right) \operatorname{rk} E^{\prime}+\tau \operatorname{rk} F^{\prime}\right) h_{E}(k)$.

On the other hand, the set of saturations $\widehat{E}^{\prime}$ of $E^{\prime}$ with $\nu \geq \mu(E)-C$ is bounded, and the set of Hilbert polynomials of such is therefore finite. For each of them, we have

$$
\mu_{\tau}^{Q}\left(\widehat{E}^{\prime}, F^{\prime}\right) \leq \mu_{\tau}^{Q}(E, F) .
$$

We may choose $K$ such that for $k \geq K$,

$$
h^{0}\left(E^{\prime} \otimes A^{k}\right) \leq h^{0}\left(\widehat{E}^{\prime} \otimes A^{k}\right)=\chi\left(\widehat{E}^{\prime} \otimes A^{k}\right) .
$$

If we have strict inequality in (5.15), then by looking at the order $k$ term we see that for $K$ chosen sufficiently large,

$$
\left(k k_{A}+M+\tau \frac{\mathrm{rk} F}{\operatorname{rk} E}\right) p\left(\widehat{E}^{\prime}, k\right)<\left(k k_{A}+M+\tau \frac{\mathrm{rk} F^{\prime}}{\operatorname{rk} E^{\prime}}\right) p(E, k) .
$$

So we assume (5.15) holds with equality. If $\operatorname{rk} F^{\prime} / \mathrm{rk} E^{\prime}=\operatorname{rk} F / \operatorname{rk} E$, then by semistability, we choose $K$ such that $p\left(\widehat{E}^{\prime}, k\right) \leq p(E, k)$. Note that if equality holds in the latter, then semistability guarantees condition (iv). 
Finally, consider the case where $\operatorname{rk} F^{\prime} / \operatorname{rk} E^{\prime} \neq \operatorname{rk} F / \operatorname{rk} E$. Since (5.15) is assumed to hold with equality, we can write

$$
\begin{aligned}
p\left(\widehat{E}^{\prime}, k\right)=p(E, k) & +\left(\tau \frac{\mathrm{rk} F^{\prime}}{\mathrm{rk} E^{\prime}}-\tau \frac{\mathrm{rk} F}{\mathrm{rk} E}\right) \frac{k^{n-1}}{(n-1) !} \\
& +C\left(\widehat{E}^{\prime}\right) \frac{k^{n-2}}{(n-2) !}+O\left(k^{n-3}\right)
\end{aligned}
$$

for some constant $C\left(\widehat{E}^{\prime}\right)$. Then we have:

$$
\begin{aligned}
\left(k k_{A}+M+\tau \frac{\mathrm{rk} F}{\mathrm{rk} E}\right) & p\left(\widehat{E}^{\prime}, k\right)=\left(k k_{A}+M+\tau \frac{\mathrm{rk} F^{\prime}}{\mathrm{rk} E^{\prime}}\right) p(E, k) \\
+ & \left(\tau \frac{\mathrm{rk} F}{\mathrm{rk} E}-\tau \frac{\mathrm{rk} F^{\prime}}{\mathrm{rk} E^{\prime}}\right) p(E, k) \\
& +\left(k k_{A}+M+\tau \frac{\operatorname{rk} F}{\operatorname{rk} E}\right)\left(\tau \frac{\mathrm{rk} F^{\prime}}{\mathrm{rk} E^{\prime}}-\tau \frac{\mathrm{rk} F}{\mathrm{rk} E}\right) \frac{k^{n-1}}{(n-1) !} \\
& +k_{A}(n-1) C\left(\widehat{E}^{\prime}\right) \frac{k^{n-1}}{(n-1) !}+O\left(k^{n-2}\right) .
\end{aligned}
$$

The order $k^{n}$ terms in the second two terms cancel. The coefficient of $k^{n-1} /(n-1)$ ! is:

$$
\begin{aligned}
&-\left\{\left(M+\tau \frac{\mathrm{rk} F}{\mathrm{rk} E}-\mu(E)\right)\right. \\
&\left.\left(\tau \frac{\mathrm{rk} F}{\mathrm{rk} E}-\tau \frac{\mathrm{rk} F^{\prime}}{\mathrm{rk} E^{\prime}}\right)-k_{A}(n-1) C\left(\widehat{E}^{\prime}\right)\right\} .
\end{aligned}
$$

Since $\tau \operatorname{rk} F / \operatorname{rk} E-\tau \operatorname{rk} F^{\prime} / \mathrm{rk} E^{\prime} \neq 0$, semistability implies this difference is positive. Therefore, it is bigger than or equal to $\tau /(\mathrm{rk} E)^{2}$. Thus (5.17) is

$$
\leq-\left\{\left(M+\tau \frac{\mathrm{rk} F}{\operatorname{rk} E}-\mu(E)\right) \frac{\tau}{(\operatorname{rk} E)^{2}}-k_{A}(n-1) C\left(\widehat{E}^{\prime}\right)\right\} .
$$

Now by the choice of $M$ in (5.9), this last expression is $\leq-1$. Since there are only finitely many possibilities for the coefficients in the $O\left(k^{n-2}\right)$ term, we can choose $K$ such for $k \geq K$ we again have

$$
\left(k k_{A}+M+\tau \frac{\mathrm{rk} F}{\mathrm{rk} E}\right) p\left(\widehat{E}^{\prime}, k\right)<\left(k k_{A}+M+\tau \frac{\mathrm{rk} F^{\prime}}{\mathrm{rk} E^{\prime}}\right) p(E, k) .
$$

This completes the proof of Lemma 5.14. 
518 Georgios Daskalopoulos, Karen Uhlenbeck and Richard Wentworth

Theorem 5.18. Fix polynomials $h_{E}$ and $h_{F}$ of degree $n$, and the parameter $\tau>0$. Choose $M$ as in (5.9). Then there exist $K, J$ such that for all $j \geq J$ the following holds: A point $\hat{q} \in \bar{Q}_{K}$ is semistable (resp. stable) with respect to the action of $S L(V)$ and the linearization determined by the Plücker embedding induced from $A^{j}$ and the line bundle $\mathcal{O}\left(K k_{A}+M, \tau\right)$ if and only if the quotient $E \rightarrow F \rightarrow 0$ associated to $\hat{q}$ has torsion-free $E$, is $\tau$-Gieseker semistable (resp. stable), and the map $V \rightarrow H^{0}\left(E \otimes A^{K}\right)$ is an isomorphism.

Proof. $(\Leftarrow)$ We first assume that $K$ has been chosen such that the conclusion of Lemma 5.14 holds. By Lemma 5.12, it suffices to find a $J$ such that for all $j \geq J$, and any subsheaf $E^{\prime} \subset E$ generated by sections of $E \otimes A^{K}$, we have

$$
\begin{array}{ll}
{\left[\left(K k_{A}+M\right) \chi\left(E \otimes A^{K+j}\right)+\tau \chi\left(F \otimes A^{K+j}\right)\right]} & h^{0}\left(E^{\prime} \otimes A^{K}\right) \\
\leq\left[\left(K k_{A}+M\right) \chi\left(E^{\prime} \otimes A^{K+j}\right)+\tau \chi\left(F^{\prime} \otimes A^{K+j}\right)\right] & h^{0}\left(E \otimes A^{K}\right)
\end{array}
$$

The set of such $E^{\prime}$ is bounded, so the set of polynomials $\chi\left(E^{\prime} \otimes A^{K+j}\right)$ and $\chi\left(F^{\prime} \otimes A^{K+j}\right)$ (in $j$ ) is finite. Comparing terms of order $j^{n}$, we see that if

$$
\begin{aligned}
{\left.\left[\left(K k_{A}+M\right) \operatorname{rk} E+\tau \operatorname{rk} F\right)\right] } & h^{0}\left(E^{\prime} \otimes A^{K}\right) \\
< & {\left.\left[\left(K k_{A}+M\right) \operatorname{rk} E^{\prime}+\tau \operatorname{rk} F^{\prime}\right)\right] h^{0}\left(E \otimes A^{K}\right), }
\end{aligned}
$$

then (5.19) holds as well, if $j$ is sufficiently large. On the other hand, if equality holds in Lemma 5.14, then (5.19) holds if and only if

$$
\begin{aligned}
\frac{\chi\left(F \otimes A^{K+j}\right)}{r \mathrm{k} E} & \leq \frac{\chi\left(F^{\prime} \otimes A^{K+j}\right)}{\mathrm{rk} E^{\prime}} \\
\Longleftrightarrow \quad \frac{\chi\left(F \otimes A^{K+j}\right)}{\mathrm{rk} F} \frac{}{\mathrm{rk} F} & \leq \frac{\chi\left(F^{\prime} \otimes A^{K+j}\right)}{\mathrm{rk} F^{\prime}} \frac{\mathrm{rk} F^{\prime}}{\mathrm{rk} E^{\prime}} \\
p \quad \Longleftrightarrow \quad(F, K+j) & \leq p\left(F^{\prime}, K+j\right),
\end{aligned}
$$

which is condition (iv) in Lemma 5.14 .

Now consider the $(\Rightarrow)$ direction. First, a slight modification of the argument in [S2] shows that, with $K$ taken sufficiently large, $E$ is torsion-free and $V \rightarrow H^{0}\left(E \otimes A^{K}\right)$ is an isomorphism. We now show that $E \rightarrow F \rightarrow 0$ is $\tau$-Gieseker semistable. Suppose not. There are several cases to consider, and we shall choose $J$ accordingly:

Case 1. Suppose $E \rightarrow F \rightarrow 0$ is $\tau$-slope-semistable, but that there exists a subsheaf $E^{\prime} \subset E$ with $p\left(E^{\prime}, j\right)=p(E, j)$ for all $j$, and $p\left(F^{\prime}, j\right)<p(F, j)$ for $j$ sufficiently large. The set of all such $E$ is bounded by Proposition 5.2, as well as the set of $E^{\prime}$. Hence, we may assume $K$ and $J$ have been chosen such 
that for all such $E, E^{\prime}, j \geq J$, we have $h^{0}\left(E^{\prime} \otimes A^{K}\right)=\chi\left(E^{\prime} \otimes A^{K}\right), p\left(F^{\prime}, j\right)<$ $p(F, j)$, and if $H=H^{0}\left(E^{\prime} \otimes A^{K}\right)$, then $h^{0}\left(F^{\prime} \otimes A^{K+j}\right)=\operatorname{dim} \pi_{2}(H \otimes W)$. Notice that the hypotheses imply rk $F^{\prime} / \mathrm{rk} E^{\prime}=\operatorname{rk} F / \operatorname{rk} E$. Thus we have

$$
\begin{gathered}
\left(\left(K k_{A}+M\right) \chi\left(E \otimes A^{K+j}\right)+\tau \chi\left(F \otimes A^{K+j}\right)\right) \operatorname{dim} H \\
=\operatorname{rk} E \operatorname{rk} E^{\prime}\left(\left(K k_{A}+M\right) p(E, K+j)+\tau \frac{\operatorname{rk} F}{\operatorname{rk} E} p(F, K+j)\right) \frac{\operatorname{dim} H}{\mathrm{rk} E^{\prime}} \\
>\operatorname{rk} E \operatorname{rk} E^{\prime}\left(\left(K k_{A}+M\right) p\left(E^{\prime}, K+j\right)+\tau \frac{\operatorname{rk} F^{\prime}}{\operatorname{rk} E^{\prime}} p\left(F^{\prime}, K+j\right)\right) \frac{\operatorname{dim} V}{\operatorname{rk} E} \\
=\left(\left(K k_{A}+M\right) \operatorname{dim} \pi_{1}(H \otimes W)+\tau \operatorname{dim} \pi_{2}(H \otimes W)\right) \operatorname{dim} V,
\end{gathered}
$$

which by Lemma 5.10 contradicts semistability.

Case 2. Suppose $E \rightarrow F \rightarrow 0$ is $\tau$-slope-semistable, but that there exists a quotient $E^{\prime \prime}$ with $\operatorname{rk} F^{\prime \prime} / \mathrm{rk} E^{\prime \prime}=\operatorname{rk} F / \operatorname{rk} E, \mu_{\tau}^{Q}\left(E^{\prime \prime}, F^{\prime \prime}\right)=\mu_{\tau}^{Q}(E, F)$, and $p\left(E^{\prime \prime}, j\right)<p(E, j)$ for large $j$. The set of all such quotients may be taken to be bounded, so we may assume $K$ has been chosen such that $p\left(E^{\prime \prime}, K\right)<$ $p(E, K)$ and $h^{0}\left(E^{\prime \prime} \otimes A^{K}\right)=\chi\left(E^{\prime \prime} \otimes A^{K}\right)$. But this now contradicts Lemma 5.13 .

Case 3. Suppose $E \rightarrow F \rightarrow 0$ is $\tau$-slope-semistable, but that there exists a quotient $E^{\prime \prime}$ with $\mu_{\tau}^{Q}\left(E^{\prime \prime}, F^{\prime \prime}\right)=\mu_{\tau}^{Q}(E, F), \mathrm{rk} F^{\prime \prime} / \mathrm{rk} E^{\prime \prime} \neq \mathrm{rk} F / \mathrm{rk} E$, and $p\left(E^{\prime \prime}, j\right)<p(E, j)$ for $j$ large. Then as in (5.16) we can write

$$
\begin{array}{r}
p\left(E^{\prime \prime}, k\right)=p(E, k)+\left(\tau \frac{\mathrm{rk} F^{\prime \prime}}{\operatorname{rk} E^{\prime \prime}}-\tau \frac{\mathrm{rk} F}{\operatorname{rk} E}\right) \frac{k^{n-1}}{(n-1) !} \\
-\frac{\operatorname{rk} E^{\prime}}{\operatorname{rk} E^{\prime \prime}} C\left(E^{\prime}\right) \frac{k^{n-2}}{(n-2) !}+O\left(k^{n-3}\right) .
\end{array}
$$

Then we have:

$$
\begin{aligned}
\left(k k_{A}+M+\tau \frac{\mathrm{rk} F}{\mathrm{rk} E}\right) & p\left(E^{\prime \prime}, k\right)=\left(k k_{A}+M+\tau \frac{\mathrm{rk} F^{\prime \prime}}{\mathrm{rk} E^{\prime \prime}}\right) p(E, k) \\
+ & \left(\tau \frac{\mathrm{rk} F}{\mathrm{rk} E}-\tau \frac{\mathrm{rk} F^{\prime \prime}}{\mathrm{rk} E^{\prime \prime}}\right) p(E, k) \\
+ & \left(k k_{A}+M+\tau \frac{\mathrm{rk} F}{\mathrm{rk} E}\right)\left(\tau \frac{\mathrm{rk} F^{\prime \prime}}{\mathrm{rk} E^{\prime \prime}}-\tau \frac{\mathrm{rk} F}{\mathrm{rk} E}\right) \frac{k^{n-1}}{(n-1) !} \\
& -(n-1) k_{A} C\left(E^{\prime}\right) \frac{\mathrm{rk} E^{\prime}}{\mathrm{rk} E^{\prime \prime}} \frac{k^{n-1}}{(n-1) !}+O\left(k^{n-2}\right) .
\end{aligned}
$$

The coefficient of $k^{n-1} /(n-1)$ ! in the last three terms is:

$$
-\left\{\left(M+\tau \frac{\mathrm{rk} F}{\mathrm{rk} E}-\mu(E)\right)\left(\tau \frac{\mathrm{rk} F}{\mathrm{rk} E}-\tau \frac{\mathrm{rk} F^{\prime \prime}}{\mathrm{rk} E^{\prime \prime}}\right)+k_{A}(n-1) C\left(E^{\prime}\right) \frac{\mathrm{rk} E^{\prime}}{\mathrm{rk} E^{\prime \prime}}\right\} .
$$


520 Georgios Daskalopoulos, Karen Uhlenbeck and Richard Wentworth

By assumption, $\mathrm{rk} F / \mathrm{rk} E-\mathrm{rk} F^{\prime \prime} / \mathrm{rk} E^{\prime \prime}>0$, so in particular,

$$
\frac{\mathrm{rk} E^{\prime \prime}}{\mathrm{rk} E^{\prime}}\left(\tau \frac{\mathrm{rk} F}{\mathrm{rk} E}-\tau \frac{\mathrm{rk} F^{\prime \prime}}{\mathrm{rk} E^{\prime \prime}}\right)=\tau \frac{\mathrm{rk} F^{\prime}}{\mathrm{rk} E^{\prime}}-\tau \frac{\mathrm{rk} F}{\mathrm{rk} E} \geq \frac{\tau}{(\mathrm{rk} E)^{2}} .
$$

By the choice (5.9) of $M$, the coefficient of $k^{n-1} /(n-1)$ ! is $\leq-\mathrm{rk} E^{\prime} / \mathrm{rk} E^{\prime \prime}<$ 0 . Thus for $K$ large, we again have a contradiction to Lemma 5.13.

Case 4. Suppose there exists a quotient $E^{\prime \prime}$ of $E$ with $\mu_{\tau}^{Q}\left(E^{\prime \prime}, F^{\prime \prime}\right)<$ $\mu_{\tau}^{Q}(E, F)$. We may assume that the $E^{\prime \prime}$ vary in a bounded family. Then we also assume $K$ has been chosen such that $h^{0}\left(E^{\prime \prime} \otimes A^{K}\right)=\chi\left(E^{\prime \prime} \otimes A^{K}\right)$. By noting the coefficient of $K^{n-1}$ and choosing $K$ larger if necessary, we may further assume that

$$
\begin{aligned}
\left(\left(K k_{A}+M\right) \mathrm{rk} E+\tau \mathrm{rk} F\right) & \chi\left(E^{\prime \prime} \otimes A^{K}\right) \\
& <\left(\left(K k_{A}+M\right) \mathrm{rk} E^{\prime \prime}+\tau \mathrm{rk} F^{\prime \prime}\right) \chi\left(E \otimes A^{K}\right) .
\end{aligned}
$$

But this now contradicts Lemma 5.13. This completes the statement about semistability. The statement about stability follows as in Case 1 above.

\subsection{Moduli space construction.}

Corollary 5.20. Let $\mathfrak{M}_{\tau}=\mathfrak{M}_{\tau}\left(h_{E}, h_{F}\right)$ denote the GIT quotient of $\bar{Q}_{K}$. Then $\mathfrak{M}_{\tau}$ is a projective scheme. The closed points of $\mathfrak{M}_{\tau}$ represent equivalence classes of $\tau$-Gieseker semistable quotients, where $(E, F) \sim\left(E^{\prime}, F^{\prime}\right)$ if $\operatorname{gr}(E, F) \sim \operatorname{gr}\left(E^{\prime}, F^{\prime}\right)$.

Proof. Since $\bar{Q}_{K}$ is projective, the first result follows from [GIT]. For the second result, note that given a semistable quotient $E \rightarrow F \rightarrow 0$, then by scaling the extensions in the Jordan-Hölder filtration Proposition 2.12, one finds as in [S2] that the orbit of $\operatorname{gr}(E, F)$ is contained in the closure of the orbit of $E \rightarrow F \rightarrow 0$. Hence, if $\operatorname{gr}(E, F)=\operatorname{gr}\left(E^{\prime}, F^{\prime}\right)$ the closures of the orbits of $(E, F)$ and $\left(E^{\prime}, F^{\prime}\right)$ intersect. Conversely, the orbit of $\operatorname{gr}(E, F)$ is closed. This follows from simplicity Proposition 2.11.

\section{References.}

[BDW] A. Bertram, G. Daskalopoulos and R. Wentworth, Gromov invariants for holomorphic maps from Riemann surfaces to Grassmanians, to appear in Journ. of AMS. 
[Br] Bradlow, S., Special metrics and stability for holomorphic bundles with global sections, J. Diff. Geom. 33 (1991), 169-214.

[BrGP] Bradlow, S. and O. Garcia-Prada, Higher cohomology triples and holomorphic extensions, preprint, 1995.

[DU] Daskalopoulos, G. and K. Uhlenbeck, An application of transversality to the topology of the moduli of stable bundles, Topology 34, No. 1 (1995), 203-215.

[Do] Donaldson, S., Antiselfdual Yang-Mills connections over complex algebraic surfaces and stable vector bundles, Proc. London Math. Soc. (3) 50 (1985), $1-26$.

[GH] Griffiths, P. and J. Harris, "Principles of Algebraic Geometry," Wiley, New York, 1978.

[GIT] Mumford, D., J. Fogarty, and F. Kirwan, "Geometric Invariant Theory," Springer, Berlin, 1994.

[Gr] Grothendieck, A., Techniques de construction et théorèmes d'existence en géométrie algébrique IV, Sem. Bourbaki 221 (1960/61).

[GS] Guillemin, V. and S. Sternberg, Geometric quantization and multiplicities of group representations, Invent. Math. 67 (1982), 515-538.

[Hi] Hitchin, N., Harmonic maps from a 2-torus to the 3-sphere, J. Diff. Geom. 31 (1990), 627-710.

[Ki] Kirwan, F., "Cohomology of Quotients in Symplectic and Algebraic Geometry," Princeton University Press, 1984.

[Ko] Kobayashi, S., "Differential Geometry of Complex Vector Bundles," Princeton University Press, 1987.

[KN] Kempf, G. and L. Ness, The length of vectors in representation spaces, Springer LNM 732 (1978), 233-242.

[KgNw] King, A. and P. Newstead, Moduli of Brill-Noether pairs, preprint, 1994.

[Kod] Kodaira, K., "Complex Manifolds and Deformation of Complex Structures," Springer, Berlin, 1986.

[Li] Li, J., Algebraic geometric interpretation of Donaldson's polynomial invariants, J. Diff. Geom. 37 (1993), 417-466.

[Ma] Maruyama, M., On boundedness of families of torsion free sheaves, J. Math. Kyoto Univ. 21-4 (1981), 673-701. 
522 Georgios Daskalopoulos, Karen Uhlenbeck and Richard Wentworth

[Nw] Newstead, P., "An Introduction to Moduli Problems and Orbit Spaces," Tata Institute, 1978.

[S1] Simpson, C., "Systems of Hodge Bundles and Uniformization," Ph. D. Thesis, Harvard University, 1987.

[S2] Simpson, C., Moduli of representations of the fundamental group of a smooth projective variety I and II, preprint, 1992.

[Th] Thaddeus, M., Stable pairs, linear systems and the Verlinde formula, Invent. Math. 117 (1994), 317-353.

[T] Tian, G., On the stability of the tangent bundles of Fano varieties, Int. J. of Math. 3 (1992), 401-413.

[U1] Uhlenbeck, K., Harmonic maps into Lie groups, J. Diff. Geom. 30 (1989), $1-50$.

[U2] Uhlenbeck, K., On the connection between harmonic maps and the selfdual Yang Mills and the sine-Gordon equation, J. Geom. Phys. 8 (1992), 283-316.

[UY] Uhlenbeck, K. and S.-T. Yau, On the existence of Hermitian-Yang-Mills connections in stable vector bundles, Comm. Pure and Appl. Math. 39 (1986), S257-S293.

RECEIVED June 9Th, 1995.

Department of Mathematics

BROWN UNIVERSITY

PROVIDENCE, RI 02912

E-MAIL ADDRESS: DASKAL@GAUSS.MATH.BROWN.EDU

Department of Mathematics

UNIVERSITY OF TEXAS

Austin, TX 78712

E-MAIL ADDRESS: UHLEN@MATH.UTEXAS.EDU

AND

Department of Mathematics

UNIVERSITY OF CALIFORNIA

IRVINE, CA 92717

E-MAIL ADDRESS: RAW@MATH.UCI.EDU 